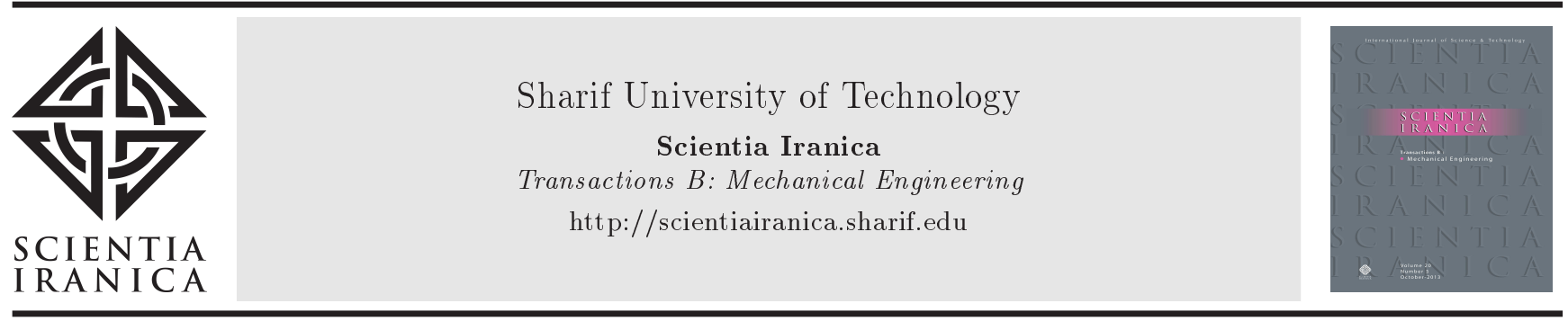

Research Note

\title{
Investigation of time-frequency analysis and transitional boundary layer over a pitching airfoil
}

\author{
H. Akhlaghi ${ }^{a}$, M.R. Soltani ${ }^{a, 1, *}$, and M.J. Maghrebi ${ }^{b}$ \\ a. Department of Aerospace Engineering, Sharif University of Technology, Tehran, Iran. \\ b. Department of Mechanical Engineering, Faculty of Engineering, Ferdowsi University of Mashhad, Mashhad, Iran.
}

Received 24 April 2019; received in revised form 18 January 2020; accepted 11 May 2020
KEYWORDS
Pitching airfoil;
Hot-film sensor;
Time-frequency
analysis;
Space-frequency
analysis;
Transition onset;
Relaminarization.

\begin{abstract}
Transitional boundary layer over a pitching airfoil at low Reynolds number $\left(\operatorname{Re}=2.7 \times 10^{5}\right)$ is experimentally investigated using space-frequency and time-frequency analyses of hot-film signals. Boundary layer events are visualized based on the spacefrequency and time-frequency plots. The precursor phenomenon for turbulent and fully separated flows is presented based on the time-frequency analysis. A new technique based on time-frequency analysis of hot-film signals is introduced to measure the transition onset and relaminarization locations. This technique functions based on the analysis of highfrequency disturbances of the measured data. Significant attention has been drawn to the spatial/temporal progression of the transition onset and relaminarization points rather than to the static values for different oscillation frequencies and amplitudes. Investigations are performed prior to, within, and beyond the static stall angle of attack conditions. The results obtained from this new technique are discussed and compared with the observations of previous investigators.

(C) 2021 Sharif University of Technology. All rights reserved.
\end{abstract}

\section{Introduction}

Boundary Layer (BL) transition plays a leading role in the performance of aerospace vehicles, wind turbines, turbomachines, etc. A detailed investigation of the location of Laminar to Turbulent Transition (LTT) could be beneficial for performance analysis and flow control of such machines. Aerodynamic designers tend to increase the range of laminar flow over the bodies to reduce drag; therefore, the transition position is considered a significant design parameter [1].

1. Present address: William E. Boeing Department of Aeronautics and Astronautics, University of Washington, Seattle, WA, USA.

*. Corresponding author. Tel.: +98 2166164601 ; Fax: +982166022731

E-mail address: msoltani@sharif.edu (M.R. Soltani)
A brief review of the literature shows that experimental detection of $\mathrm{BL}$ events has been traditionally performed using different approaches based on the surface pressure data, hot-wire and hot-film data, acoustic data, and flow visualization techniques. The hot-film sensors have been primarily used as a pointwise skin friction indicator [2]. Hot-film anemometry is a relatively nonintrusive technique that allows for transition detection with a high temporal resolution. The measurement principle is based on the convective heat transfer from the heated element to the fluid. The hot-film anemometry was successfully employed to investigate BL events in both steady and unsteady conditions. Interpreting the BL events based on the raw data of hot-film outputs in the form of voltages or Quasi-Wall Shear Stress (QWSS) values is the most common method. Stack et al. [3,4] developed a technique based on hot-film output voltages to detect separation points. In their technique, the adjacent hot- 
film sensors, located before and after the separation point, showed a $180 \mathrm{deg}$ phase-shift in their output signals. In their proposed technique, separation and reattachment points could be detected. This technique was applicable to both steady and unsteady airfoils. However, it was more effective in unsteady airfoils, where separation and reattachment points were more pronounced mainly due to the body motion. Hodson et al. [5] proposed a formula to determine QWSS parameter through hot-film voltages. Using this formula, one could qualitatively determine the distribution of wall shear stress along the BL using hot-film measurements. Stack et al. [3] measured the frequency of the Tollmien-Schlichting (TS) waves based on the frequency analysis of the hot-film data. The field contours of the hot-film voltages were employed to visualize the BL events such as Laminar Separation Bubble (LSB), dynamic stall vortex, transition and relaminarization, and reattachment processes over a pitching airfoil [6,7]. Lee et al. [8,9] detected BL events over a pitching airfoil using the raw data measured by closely spaced multiple hot-film sensors. Rudmin et al. $[10,11]$ measured the separation and transition locations for a pitching airfoil based on the crosscorrelation and spectra of the hot-film data. Gardner et al. [12-14] investigated laminar, transitional, and turbulent BL over a pitching airfoil using the hot-film raw voltages.

Following the previous experimental measurements of the BL events from the hot-film sensors, the present study aims to investigate the unsteady transitional BL over a pitching airfoil using timespace and time-frequency interpretation of the hotfilm outputs which, to the authors' knowledge, has not been extensively reported in the literature survey. Moreover, a new Root-Mean-Squared (RMS) parameter was defined based on the time-frequency analysis of the hot-film signals that could be used for detecting both unsteady transition and relaminarization onset. This research followed the previous study of Soltani et al. [15-19] on explaining the aerodynamic behavior of the current airfoil model in steady and unsteady conditions.

\section{Experimental setup}

All experiments were conducted in a low-speed wind tunnel, an open-circuit tunnel with a rectangular test section of $80 \times 100 \times 200 \mathrm{~cm}^{3}$. The inlet of the tunnel has three large anti-turbulence screens and a honeycomb in its settling chamber to reduce the tunnel turbulence level and produce a uniform flow in its test section. Measurement inaccuracies of the freestream parameters are listed in Table 1.

A model with the chord of $25 \mathrm{~cm}$ and span of $80 \mathrm{~cm}$ was employed in this investigation. The model was constructed of fiberglass with the measured accuracy of $\pm 0.1 \mathrm{~mm}$. To measure the pressure distribution over the surface of the model, 63 pressure taps were used and congested at the leading edge (Figure 1). The surface pressure was measured using differential pressure sensors whose pressure ranged from 0.075 to 1.0 psi and the maximum full span error was $0.15 \%$. Sixteen hot-film sensors were located on the upper surface of the airfoil at $0.204 \leq x / c \leq 0.752$ (see Table $2)$. The employed hot-film sensor is manufactured by Senflex ${ }^{\circledR}$, model SF9902, which is a single-element hot film sensor on a $120 \mathrm{~mm} \times 20 \mathrm{~mm}$ substrate. The substrate is 2-mil thick Upilex ${ }^{\circledR}$ S polyimide film. Hotfilm sensors were installed over the model surface using a suitable adhesive. The adhesive has a thickness of 3 mil designed for the environment temperature. The lead wires were soldered to the ends of the sensors and carried to the connector. The length of the wire from the surface to the Constant Temperature Anemometer (CTA) was about 4 meters. Uncalibrated hot-film data were also used to define the QWSS and provide semi-quantitative information about the state of the BL development. Hot film outputs were obtained by DANTEC Constant Temperature Anemometer (CTA). All hot films were sampled at a frequency of $5 \mathrm{kHz}$. An overheat ratio of 0.9 was selected for all hot films in

Table 1. Freestream flow measurement inaccuracies (\%).

\begin{tabular}{ccccc}
\hline $\boldsymbol{\Delta} \boldsymbol{T} / \boldsymbol{T}$ & $\boldsymbol{\Delta} \boldsymbol{P} / \boldsymbol{P}$ & $\boldsymbol{\Delta} \boldsymbol{\rho} / \boldsymbol{\rho}$ & $\boldsymbol{\Delta} U_{\infty} / U_{\infty}$ & $\boldsymbol{\Delta} R_{\boldsymbol{e}} / R_{\boldsymbol{e}}$ \\
\hline $\mathbf{0 . 0 3 3}$ & 1.421 & 0.035 & 2.009 & 2.010 \\
\hline
\end{tabular}

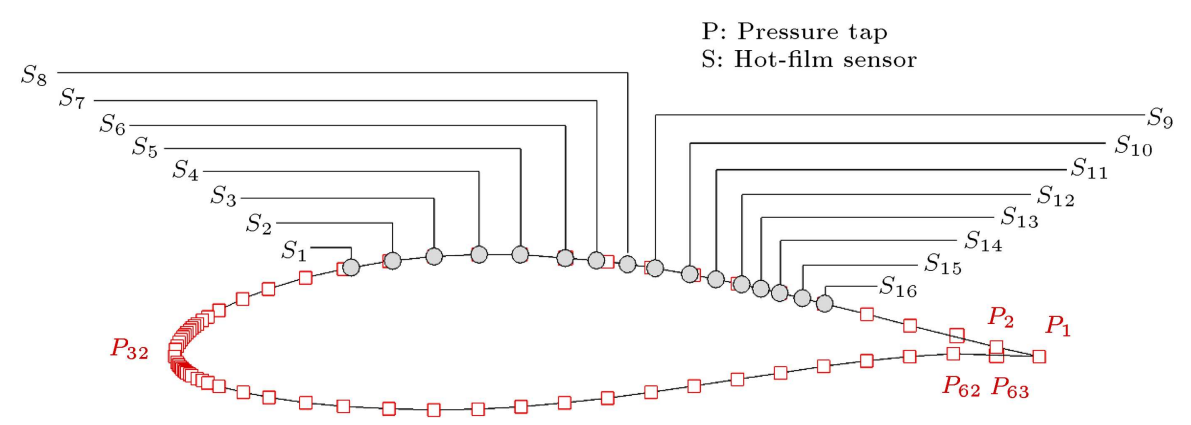

Figure 1. Schematic view of the airfoil model with the pressure taps and hot-film sensors. 


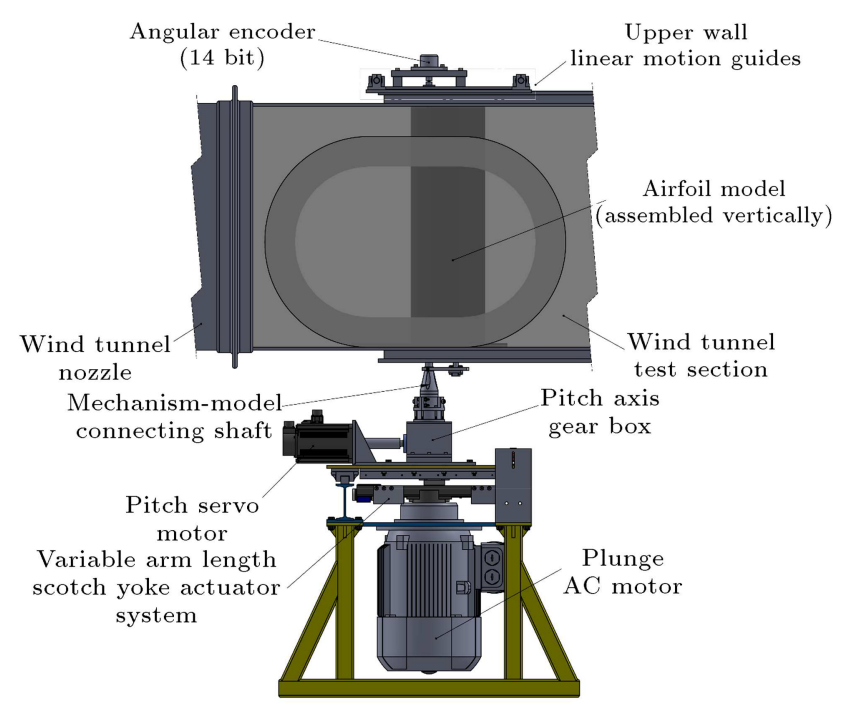

Figure 2. Schematic view of the airfoil model installation configuration on wind turbine blade motion simulator in the wind tunnel test section.

Table 2. Locations of the hot-film sensors.

\begin{tabular}{cccc}
\hline Sensor ID & $\boldsymbol{x} / \boldsymbol{c}$ & Sensor ID & $\boldsymbol{x} / \boldsymbol{c}$ \\
\hline$S_{1}$ & 0.204 & $S_{9}$ & 0.556 \\
$S_{2}$ & 0.252 & $S_{10}$ & 0.596 \\
$S_{3}$ & 0.300 & $S_{11}$ & 0.626 \\
$S_{4}$ & 0.352 & $S_{12}$ & 0.656 \\
$S_{5}$ & 0.400 & $S_{13}$ & 0.678 \\
$S_{6}$ & 0.452 & $S_{14}$ & 0.700 \\
$S_{7}$ & 0.488 & $S_{15}$ & 0.726 \\
$S_{8}$ & 0.524 & $S_{16}$ & 0.752 \\
\hline
\end{tabular}

order to obtain a much more appropriate response and avoid film defection. To eliminate white noise from the signals, a low-pass filter was employed.

A mechanism for pitching, plunging, and combined pitching-plunging oscillating motions capable of changing the airfoil sinusoidal motions at different amplitudes and frequencies was specially designed for the present experiment. Figure 2 shows a schematic of the aforementioned mechanism, according to which the accuracy of pitching actuation is $0.0013 \mathrm{deg}$. The oscillation frequency was measured with an accuracy rate of $\pm 0.02 \mathrm{~Hz}$. The Angles Of Attack (AOA) were measured using a 14-bit encoder with the accuracy of 0.022 deg. Different sources of error that may cause erroneous measured data are non-uniform at the flow and turbulence levels, AOA setting, pressure sensors, airfoil model construction, encoder, A/D board, hotfilms, pressure tap locations on the airfoil, wall effect, model blockage, etc. An extensive error analysis was carried out to validate the obtained data.

\section{Physical description}

To a low Reynolds number flowing over an airfoil was assigned an LTT zone over the suction side of the airfoil after an LSB. The LTT zone is somewhere between the laminar separation and turbulent reattachment points. In fact, transition occurs when there is an increase in the pressure in the $\mathrm{BL}$ due to the formation of a separation bubble [20]. The reattachment process is initiated due to a turbulent wedge that spreads from a point in the detached shear layer. For an oscillating airfoil, the BL events vary during the oscillation cycle. Figure 3 shows the output voltages for hot-film sensors installed over the upper surface of the airfoil for a pitch oscillation with a frequency of $1.02 \mathrm{~Hz}$, an amplitude of $2 \mathrm{deg}$, and an angle of attack of $6 \mathrm{deg}$. BL events from laminar to turbulent flow could be observed from the figure. As shown, the mean values for the output of each sensor vary with time according to the motion of the body. The reason why this variation happens is that each sensor experiences different values of shear stress with time as the AOA changes. In the laminar flow region, i.e., signals (a) and (b) in Figure 3 , both sensors have low voltage amplitudes. As the BL becomes unstable, the periodic turbulent bursts start to appear with an associated increase in the hotfilm output amplitudes, as seen in signal (c). The initial appearance of the peak values corresponds to the transition onset. The amplitude reaches its maximum at peak transition (d). In the turbulent region, there is

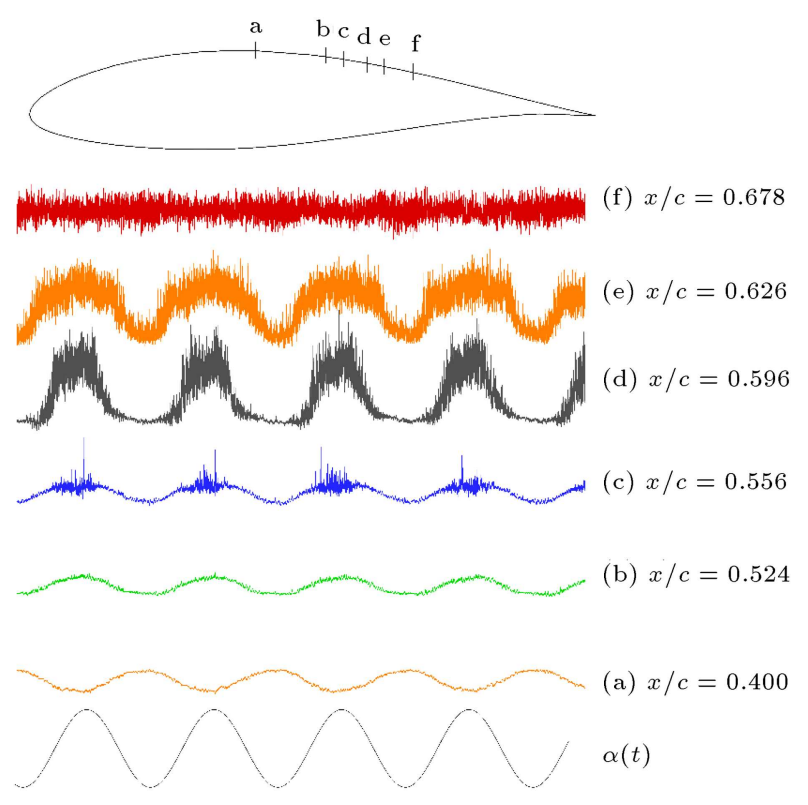

Figure 3. Hot-film signals for a pitch oscillation with $\alpha=(6 \pm 2)$ deg. 
a slight decrease in the amplitude level; see signals (e) and (f) in Figure 3.

\section{Results and discussions}

In the present section, the experimental results obtained from the hot-film sensors are reported and discussed. The results from hot-film sensors are investigated, interpreted, and classified in four different categories including hot-film voltages, spacefrequency analysis, time-frequency analysis, and transition/relaminarization onset detection based on the time-frequency analysis. The mean AOA varied from zero to ten degrees covering all ranges of the attached and separated flows corresponding to before, within, and beyond the static stall AOA $\left(\alpha_{s s}\right)$ of the present airfoil. It should be noted that the static stall AOA for the current airfoil is about $11 \mathrm{deg}$. for the present test condition. Two different oscillation frequencies $\left(f_{0}\right)$ of $1.02 \mathrm{~Hz}$ and $2.55 \mathrm{~Hz}$ were taken into account that corresponded to the reduced frequency values $(\kappa=$ $\left.\pi f_{0} c / U_{\infty}\right)$ of 0.04 and 0.1 , respectively. The velocity of the wind tunnel was set to $20 \mathrm{~m} / \mathrm{s}$. The airfoil pitch axis was located on the chord and at a distance of $c / 4$ from the leading edge. The instantaneous AOA changes during the oscillation cycle as $\alpha(t)=$ $\alpha_{m}+\alpha_{a} \sin \left(2 \pi f_{0} t\right)$. Figures 4 and 5 show the hotfilm output voltages for different mean AOAs and for oscillation amplitudes of 2 and $5 \mathrm{deg}$. The trends for AOA are given by thick gray lines in these figures. Time signals of the hot-film sensors reveal the flow characteristics over the model surface. For the cases with $\alpha_{m}=0 \mathrm{deg}$ and $\alpha_{m}=6 \mathrm{deg}$, the output signals of the sensors placed in the upstream locations are smooth and sinusoidal in shape, indicating that these sensors are located in the laminar region.

Moreover, the voltage changes for these sensors are small during the oscillation cycle, mainly due to the fact that for these sensors, variations in the wall shear stress with respect to the AOA are small. As mentioned earlier, the spatial and temporal domains of the transitional and turbulent flows over the surface of the model could be observed from the output signals of hot films. Some hot-film sensors experience all flow regimes including laminar, transitional, and turbulent flows in an oscillation cycle. According to Stack et al. [3], the adjacent hot-film sensors located before and after the separation points have a $180 \mathrm{deg}$ phase shift in their output time signals. For example, in Figure 5(b), for the pitching case with a reduced frequency of 0.1 oscillating around a mean AOA of 0 deg with an amplitude of $5 \mathrm{deg}$, flow separation in the laminar flow region or LSB onset first occurs between the hot-film sensors located at $x / c=0.524$ and $x / c=0.556$. Moreover, in this case, the LSB ends somewhere between $x / c=0.656$ and $x / c=0.678$, Figure $5(\mathrm{~b})$. Separation in the turbulent flow could also be detected by the 180 deg phase-shift technique. For instance, in Figure 5 (b), for the pitching case with a reduced frequency of 0.1 , oscillating around a mean AOA of $10 \mathrm{deg}$ with an amplitude of $5 \mathrm{deg}$, turbulent flow separation could be first detected somewhere between $x / c=0.204$ and $x / c=0.252$. The most downstream location for the turbulent flow separation in the mentioned case occurs between $x / c=0.626$ and $x / c=0.678$. As observed in Figures 4 and 5, both separation and reattachment points vary with the instantaneous changes of AOA, mean AOA, and reduced frequency. Higher mean AOA and higher reduced frequency could take both separation and reattachment points upstream.

In the following section, the space-frequency analysis of the hot-film outputs is elaborated. The proposed method along with an in-depth derivation of the equations used will be explained. This method will be employed to analyze the present experimental data. The un-calibrated hot-film data are used to provide semi-quantitative information about the state of the BL development over the airfoil surface. The QWSS $(\tau)$ is defined as follows [5]:

$$
\tau=\left(\frac{E^{2}-E_{0}^{2}}{E_{0}^{2}}\right)^{3},
$$

where $E$ is the instantaneous hot-film voltage and $E_{0}$ is the hot-film voltage in the zero-flow condition. By using the Fourier series, a measured QWSS signal could be stated as follows:

$$
\tau(x)=\sum_{n=0}^{\infty} A_{n} \sin \left(2 \pi f_{n} x\right),
$$

where $A_{n}$ denotes wave amplitude of the signal for each frequency. The space-frequency analysis of the QWSS parameter could be performed by plotting the contours of the wave amplitudes in the space-frequency domain, as shown in Figures 6 and 7 . The colors correspond to the values of $\log \left(A_{n}\right)$. The results have been reported for different pitching cases with different oscillation frequencies, mean AOAs, and oscillation amplitudes. Different events of the BL could be identified from these space-frequency plots. The TS waves are characterized by high energy frequencies before transition occurs, as marked in Figures 6 and 7. The onset of the LSB could also be captured from these figures where amplitudes are minimum. At the separation point, the shear stress reaches its minimum value. Laminar, transitional, and turbulent regions of BL could be easily detected from the space-frequency plots. Laminar and turbulent flow regions are denoted by $L$ and $T$ in Figures 6 and 7. The transitional region occurs after LSB onset and it is indicated by large amplitudes with a nonuniform frequency distribution. The transitional region 


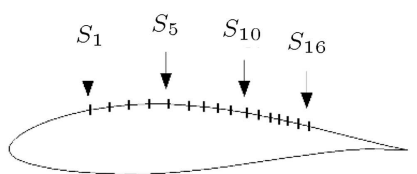

$\alpha_{m}=0^{\circ}$

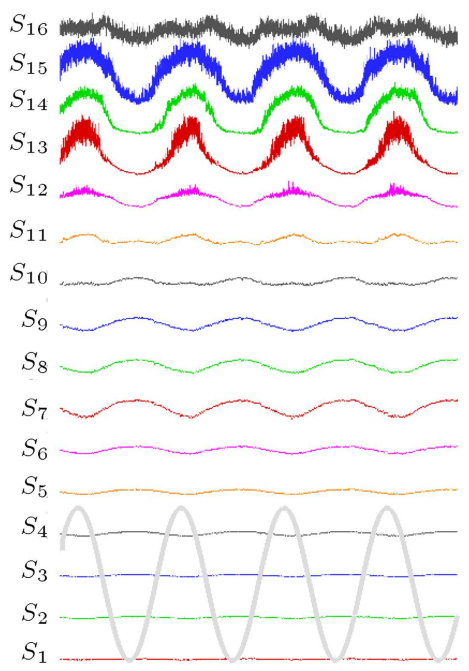

$\alpha_{m}=0^{\circ}$

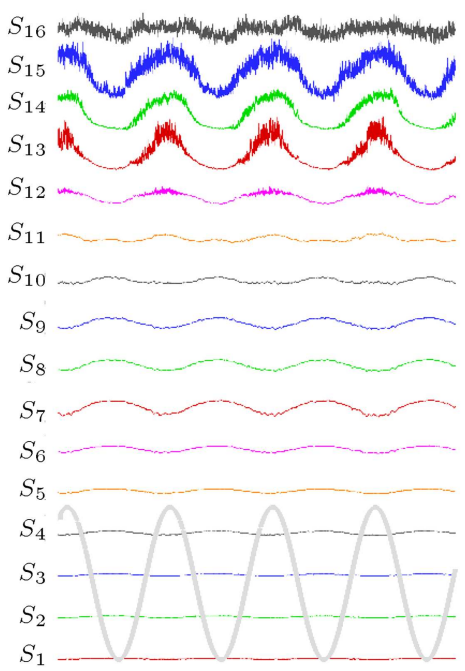

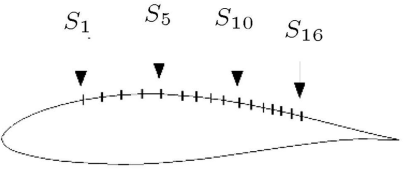

$\alpha_{m}=6^{\circ}$

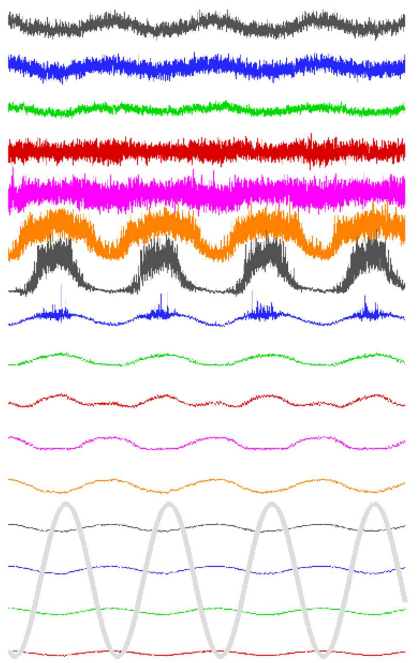

(a) $\kappa=0.04$

$\alpha_{m}=6^{\circ}$
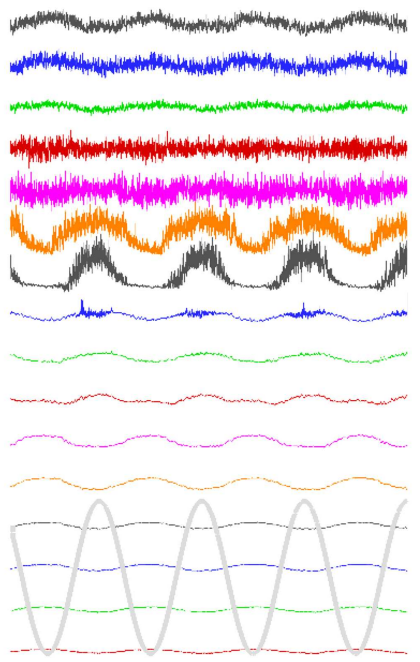

(b) $\kappa=0.10$

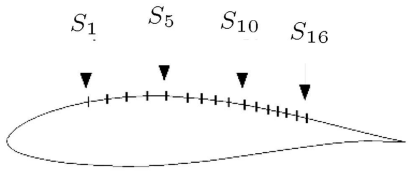

$\alpha_{m}=10^{\circ}$

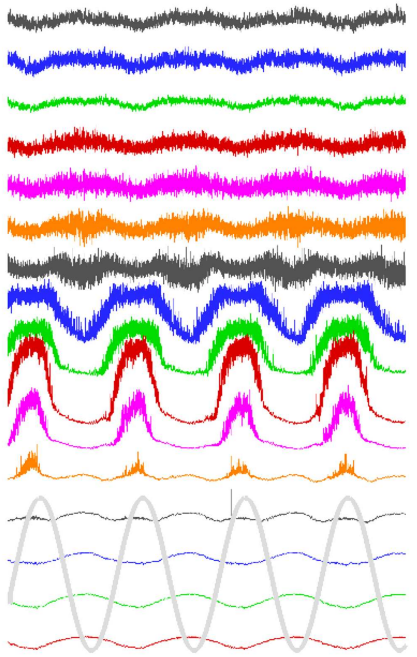

$\alpha_{m}=10^{\circ}$

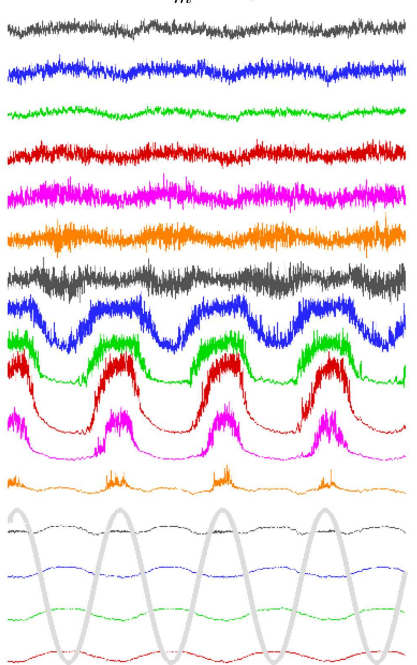

Figure 4. Hot-film output signals for oscillations with an amplitude of 2 deg.

is followed by the turbulent region with a very high amplitude and a uniform distribution in the frequency. At a larger oscillation amplitude, Figure 7 , the regions of transitional and turbulent flows are larger. For oscillations around the mean angle of $10 \mathrm{deg}$, i.e., oscillation within and beyond $\alpha_{s s}$ for this airfoil, all hot-film sensors experience turbulent flow. However, because of the unsteady motion of the model, separation points and transitional flow regions vary with time, leading to complex realization of these phenomena in the spacefrequency plots.

For an oscillating body or even for a static model in the presence of transitional flow which is unsteady, the signal of hot-film output is non-stationary. For a non-stationary signal, the statistical properties (such as mean, variance, spectral content, etc.) all change with time. As stated by Trapier et al. [21], this type of signal should be studied using spectral descriptions as 


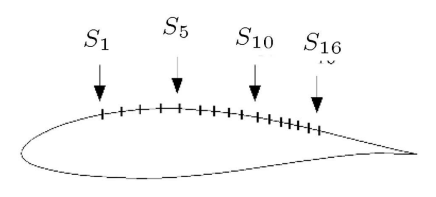

$\alpha_{m}=0^{\circ}$
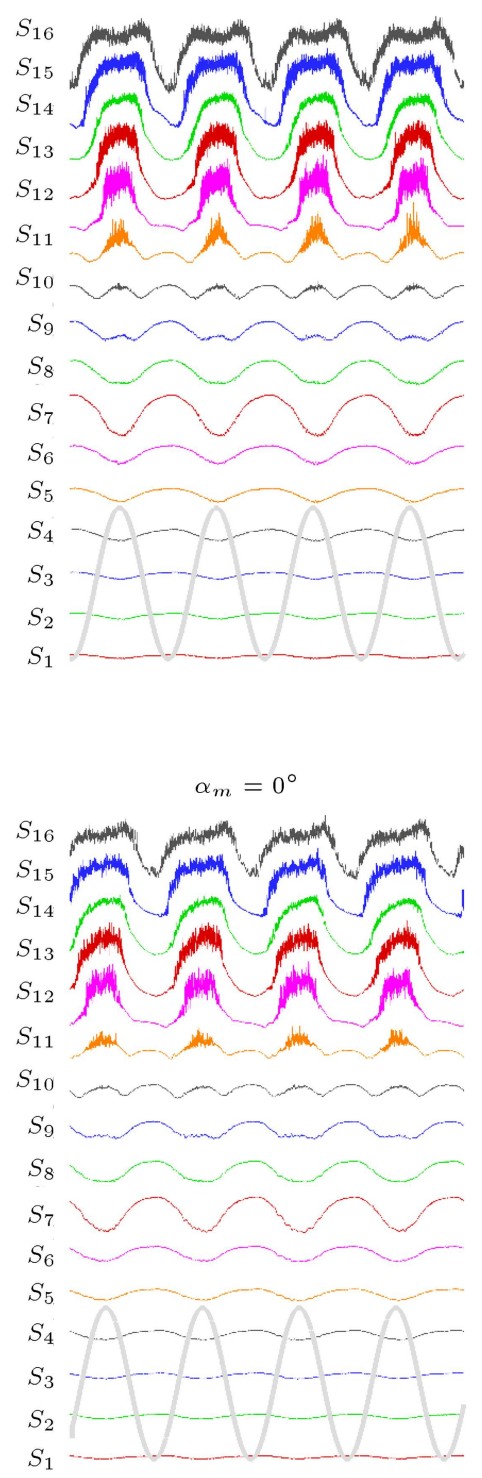

Figure 5. Hot-film output signals for oscillations with an amplitude of 5 deg.

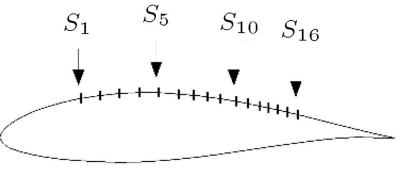

$\alpha_{m}=6^{\circ}$

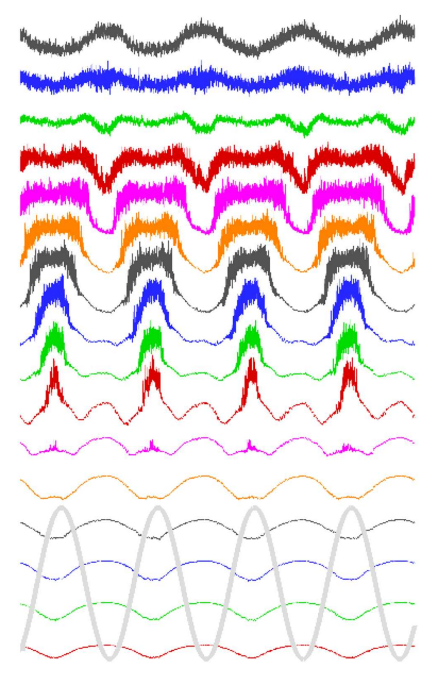

(a) $\kappa=0.04$

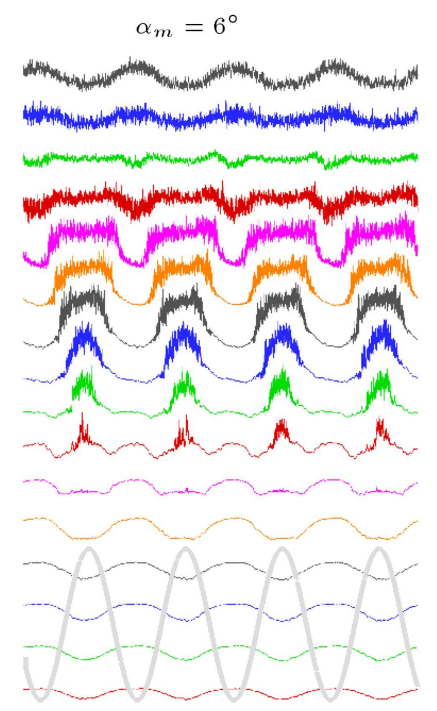

(b) $\kappa=0.10$
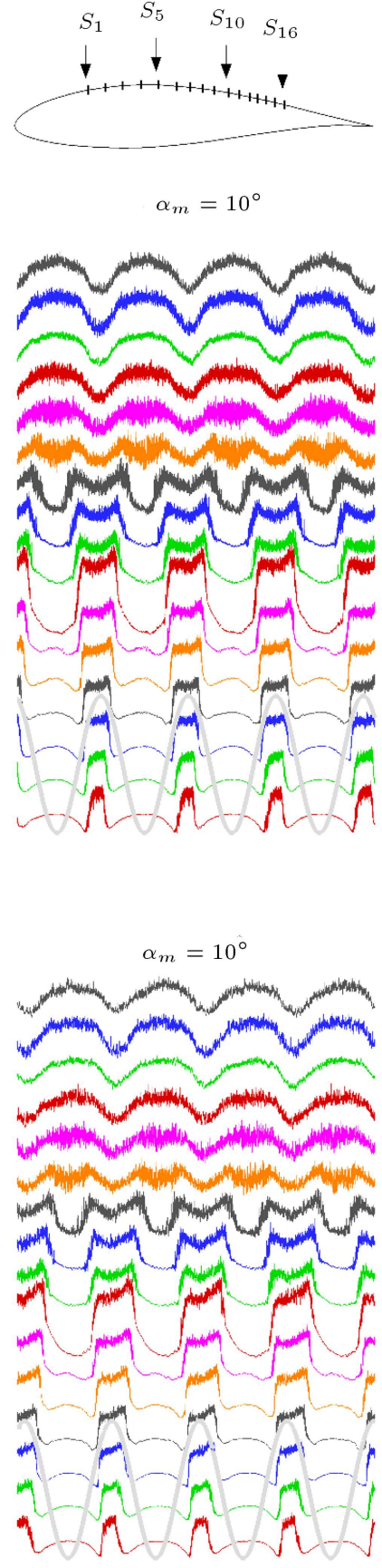

functions of both time and frequency. In this study, the BL events were investigated using spectrogram plots for hot-film sensors. Similar investigations, including that of Soltani et al., have been previously aimed at interpreting the data obtained from pressure transducers for intake analysis [22]. The idea behind the spectrogram was to apply FT to a windowed part of the signal with a finite duration instead of applying the entire signal. More details of the procedure can be found in [21]. Figure $8(\mathrm{a})-(1)$ shows the time-frequency analysis in the form of spectrogram plots obtained from the hotfilm outputs in different oscillation cases. The vertical axis corresponds to the frequencies of disturbances that range from zero up to $2.5 \mathrm{kHz}$. The horizontal axis indicates time equal to two oscillation cycles. The spectrogram plot represents the contour of the wave amplitudes for a hot-film output signal in the timefrequency domain. Each contour is reported for a specific spatial point that corresponds to a specific hotfilm sensor. The variations of the BL events with 

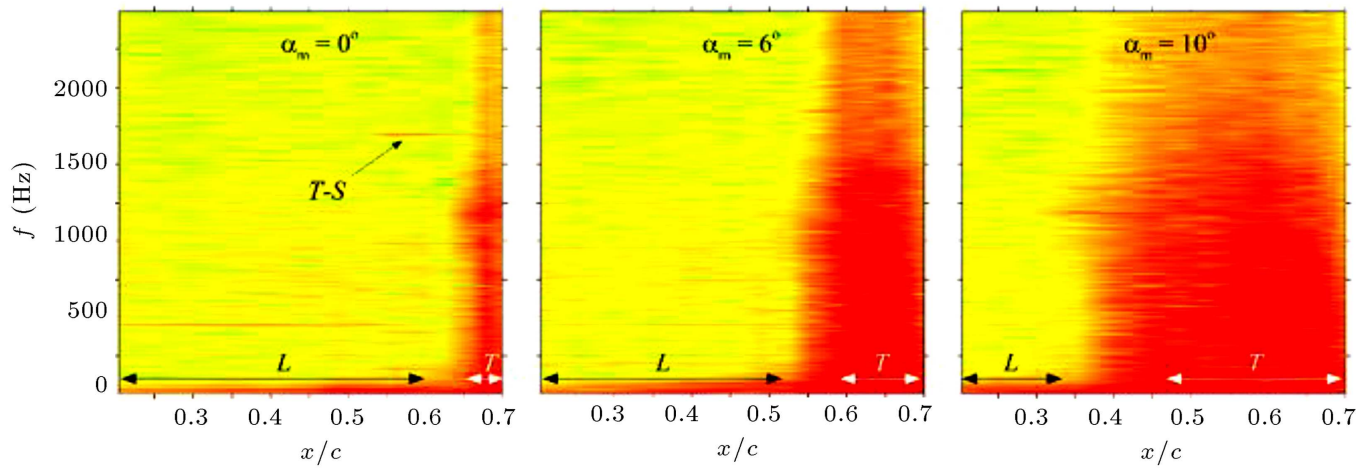

(a) $\kappa=0.04$
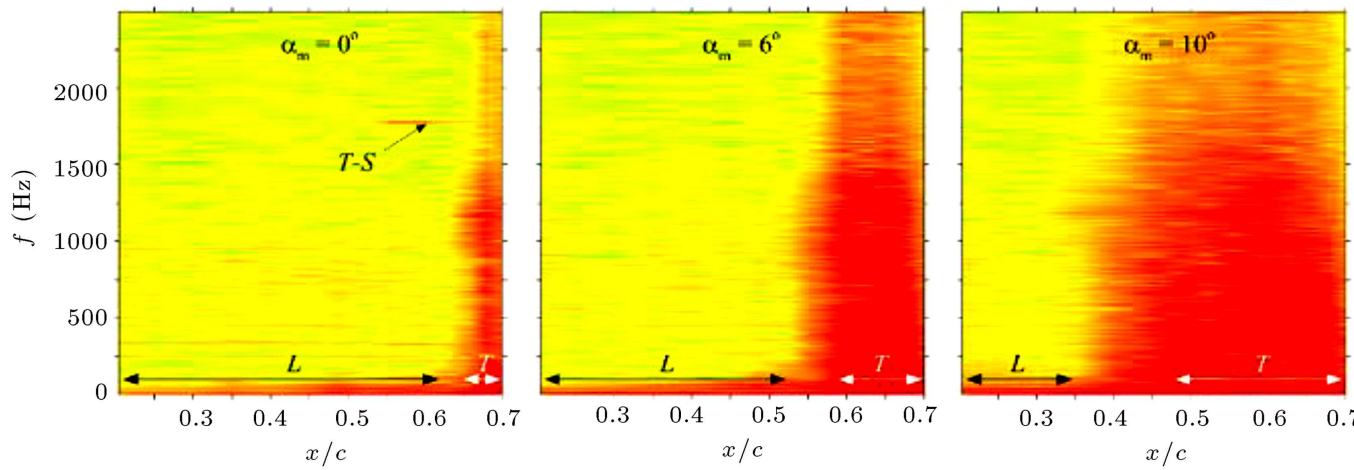

(b) $\kappa=0.10$

Figure 6. Space-frequency plots for oscillations with an amplitude of 2 deg.
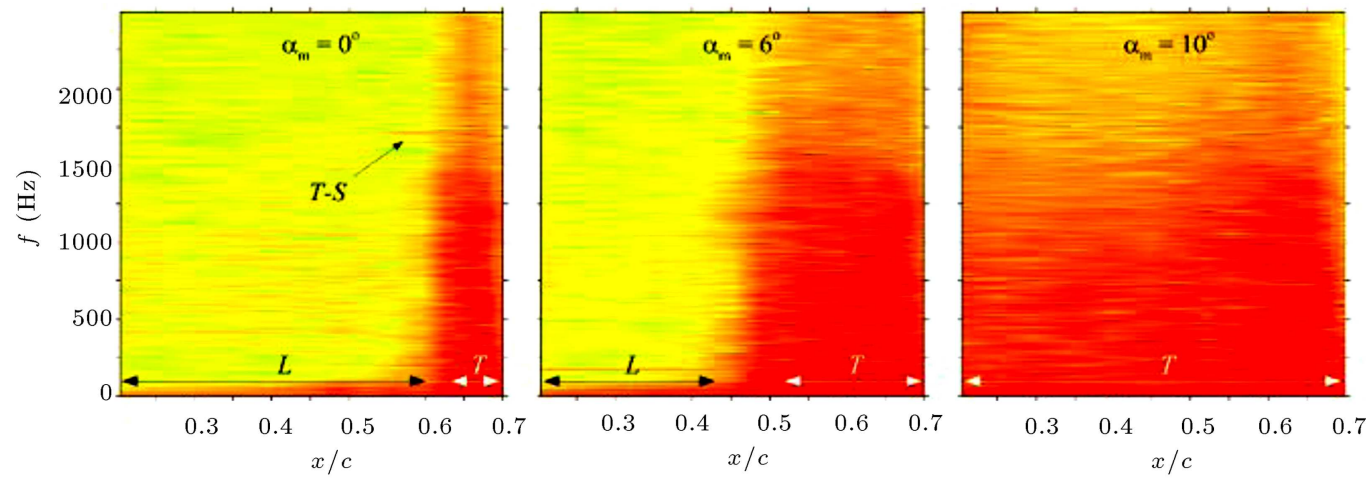

(a) $\kappa=0.04$
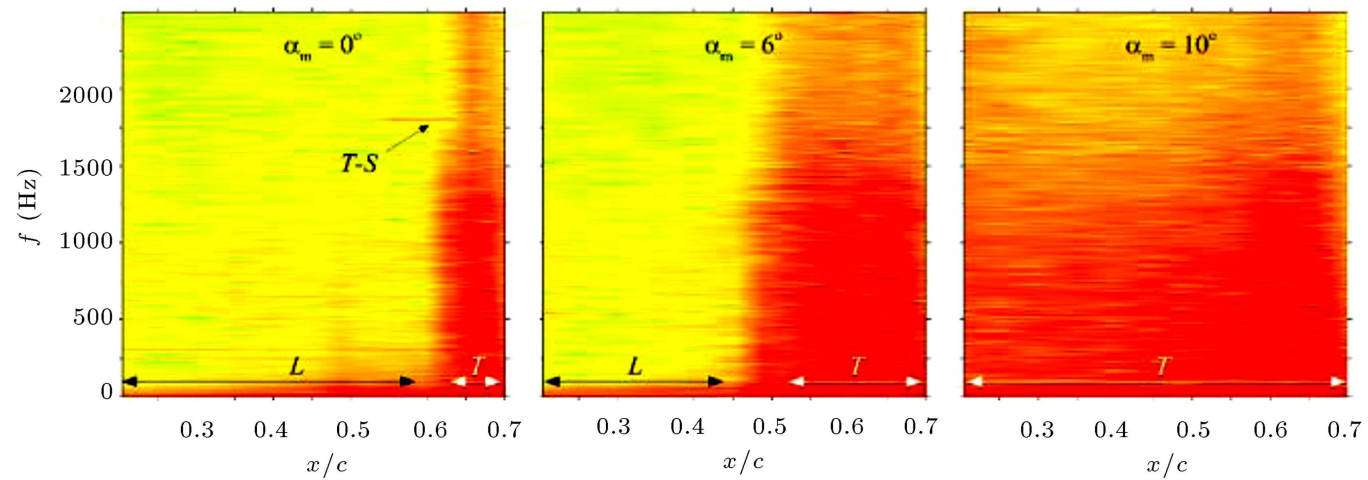

(b) $\kappa=0.10$

Figure 7. Space-frequency plots for oscillations with an amplitude of $5 \mathrm{deg}$. 

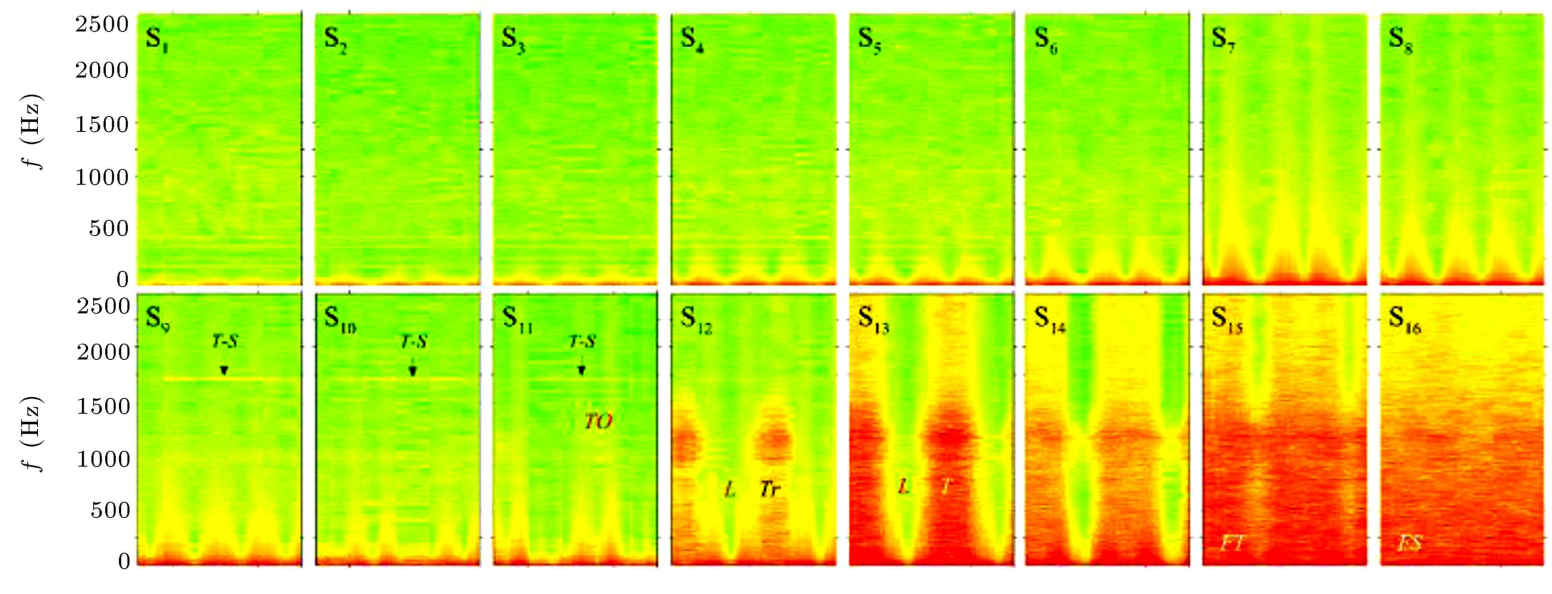

(a) $\kappa=0.04, \alpha_{m}=0 \mathrm{deg}$, and $\alpha_{a}=2 \mathrm{deg}$
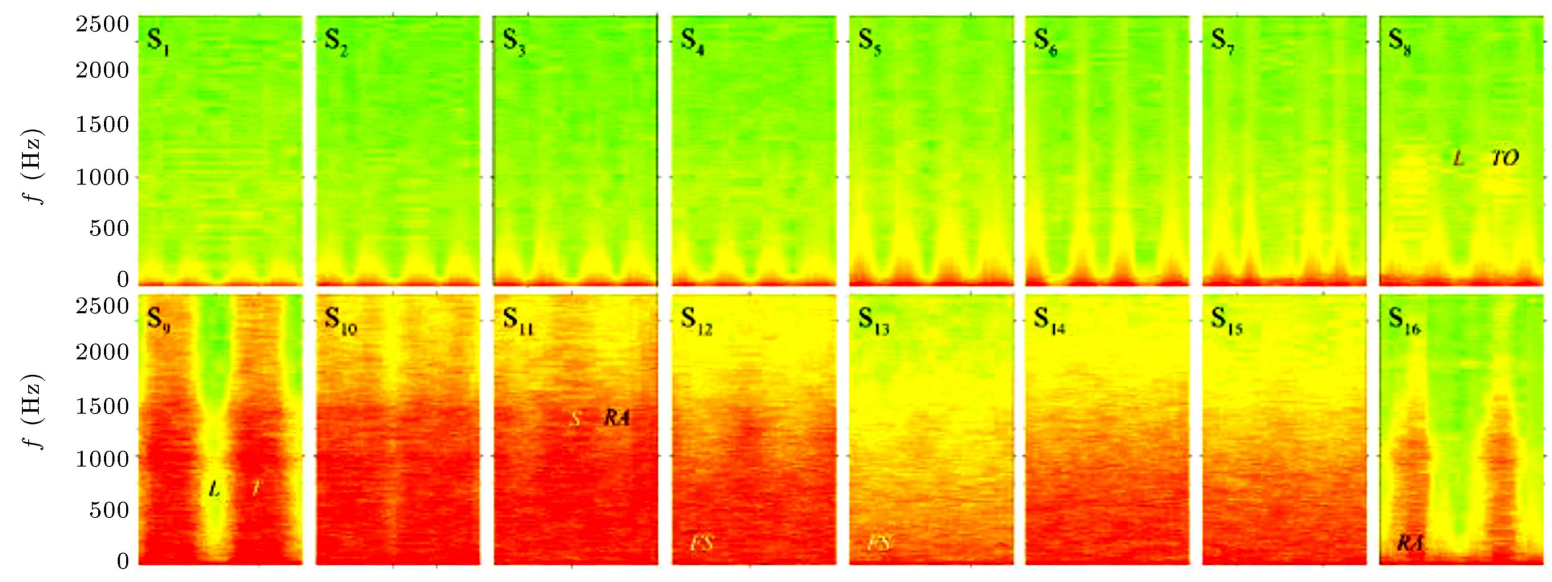

(b) $\kappa=0.04, \alpha_{m}=6 \mathrm{deg}$, and $\alpha_{a}=2 \mathrm{deg}$
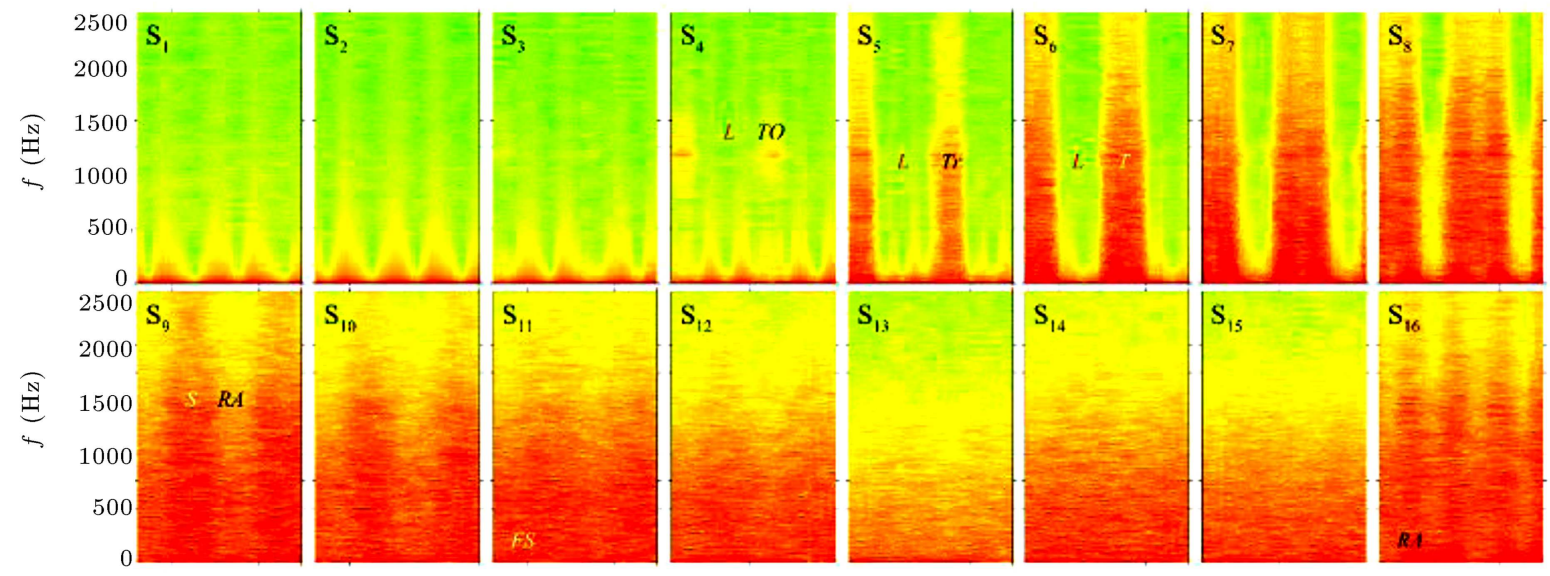

(c) $\kappa=0.04, \alpha_{m}=10 \mathrm{deg}$, and $\alpha_{a}=2 \mathrm{deg}$

Figure 8. Effects of oscillation parameters on the spectrogram of the hot-film outputs.

time are observed in the spectrogram plots. The TS waves could be detected as the dominant highfrequency waves in the spectrogram plots for the sensors located in the laminar flow region, as seen in Figure 8(b). The laminar flow regions are domains with low-amplitude, yet high-frequency, waves.
These domains are marked by $L$ in the spectrogram plots, as seen in Figure 8(b). Turbulent flow regions are visualized as the spatial/temporal domains with high-amplitude high-frequency waves. These domains are marked by $T$ in Figure $8(\mathrm{a})-(\mathrm{l})$. According to Figure $8(\mathrm{a})$, the transitional flow domains have high- 

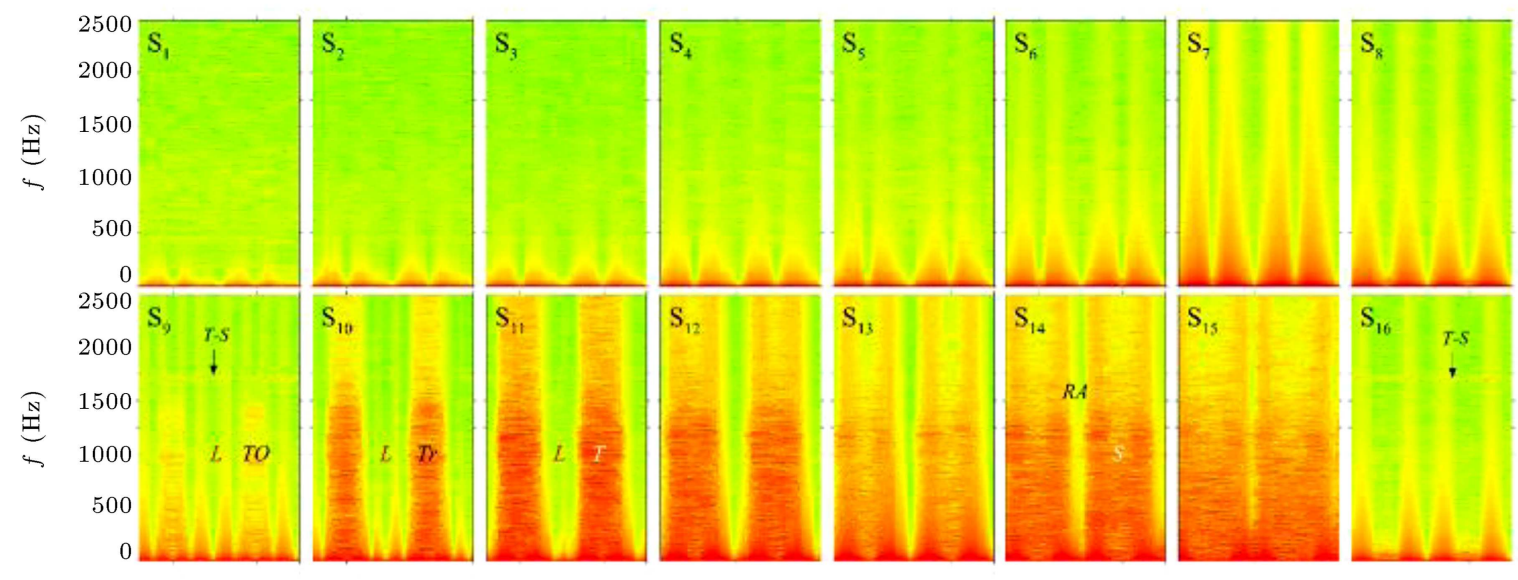

(d) $\kappa=0.04, \alpha_{m}=0 \mathrm{deg}$, and $\alpha_{a}=5 \mathrm{deg}$
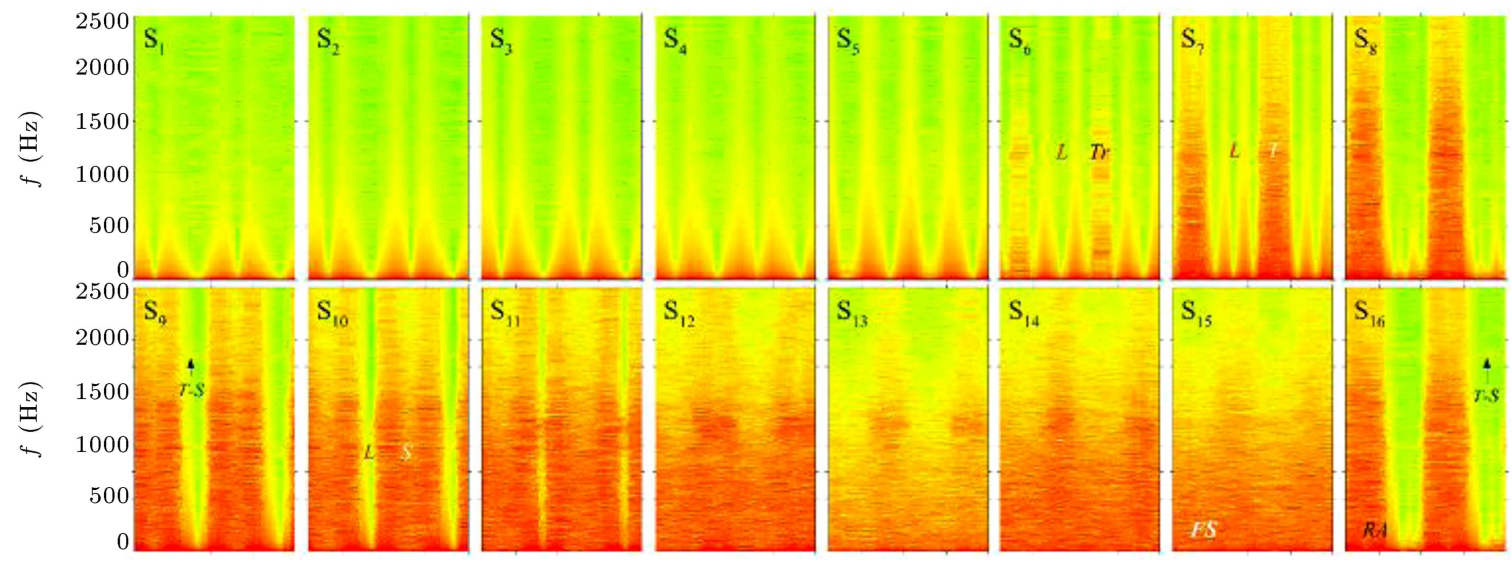

(e) $\kappa=0.04, \alpha_{m}=6 \mathrm{deg}$, and $\alpha_{a}=5 \mathrm{deg}$
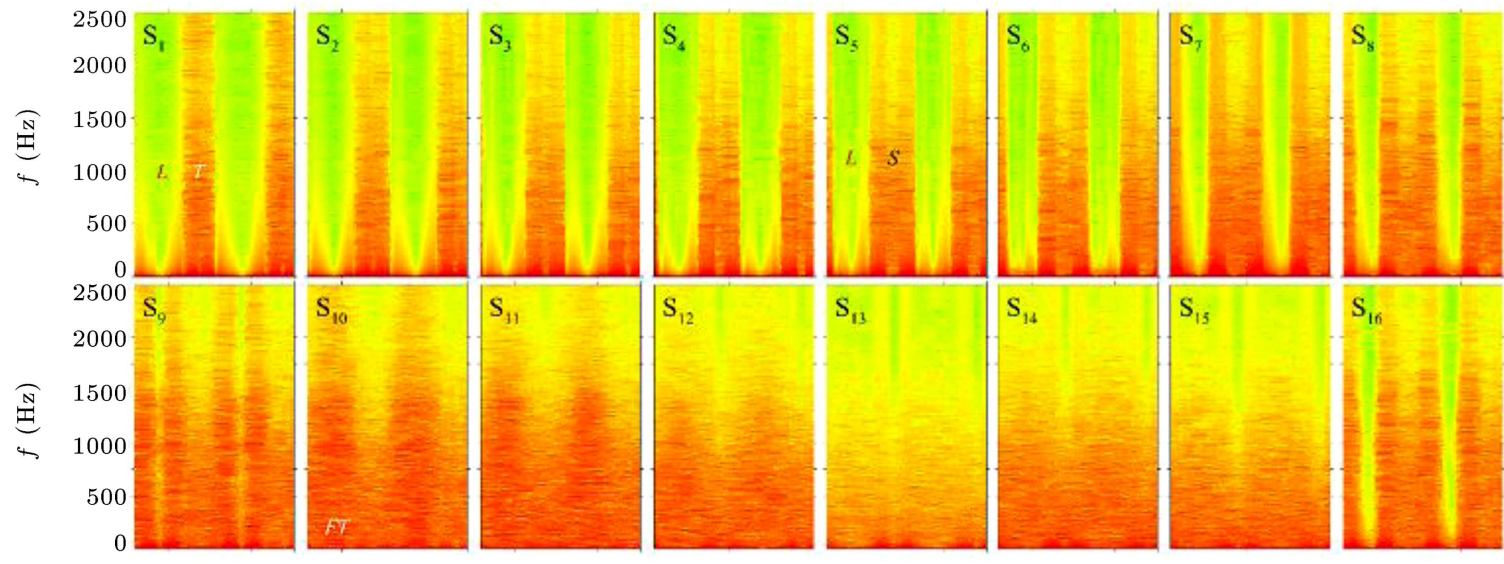

(f) $\kappa=0.04, \alpha_{m}=10 \mathrm{deg}$, and $\alpha_{a}=5 \mathrm{deg}$

Figure 8. Effects of oscillation parameters on the spectrogram of the hot-film outputs (continued).

amplitude mid-frequency waves and are marked by $T r$. As observed in Figure 8(a)-(c), the hot-film sensor should be located in a place where it faces the transition onset during a portion of the oscillation cycle marked by TO. Separated flow zones are domains with highamplitude high-frequency waves that are continuous and do not change in a certain period of time. These domains are denoted by $S$ in the spectrogram plots. The separated flow can be reattached when the AOA decreases in the oscillation cycle. The reattached flows are marked by RA, as shown in Figure 8(d). In Figure 8(e), the sensors located in the downstream 

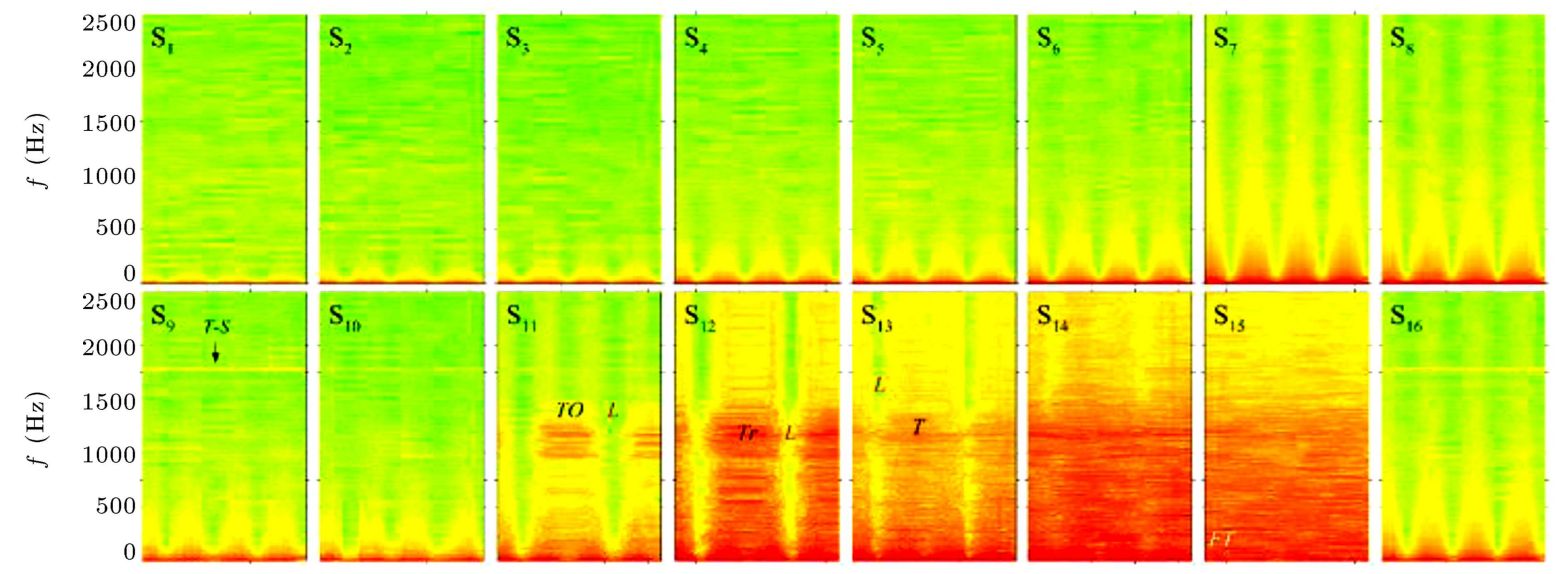

(g) $\kappa=0.1, \alpha_{m}=0 \mathrm{deg}$, and $\alpha_{a}=2 \mathrm{deg}$
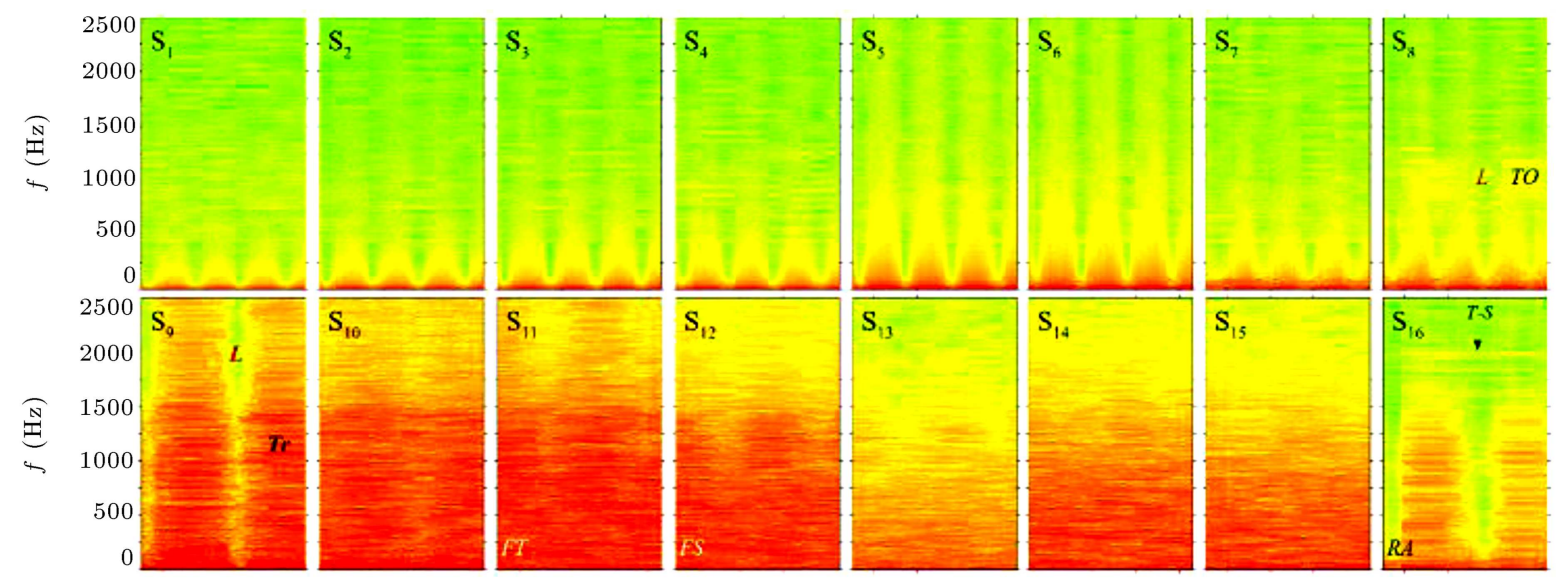

(h) $\kappa=0.1, \alpha_{m}=6 \mathrm{deg}$, and $\alpha_{a}=2 \mathrm{deg}$
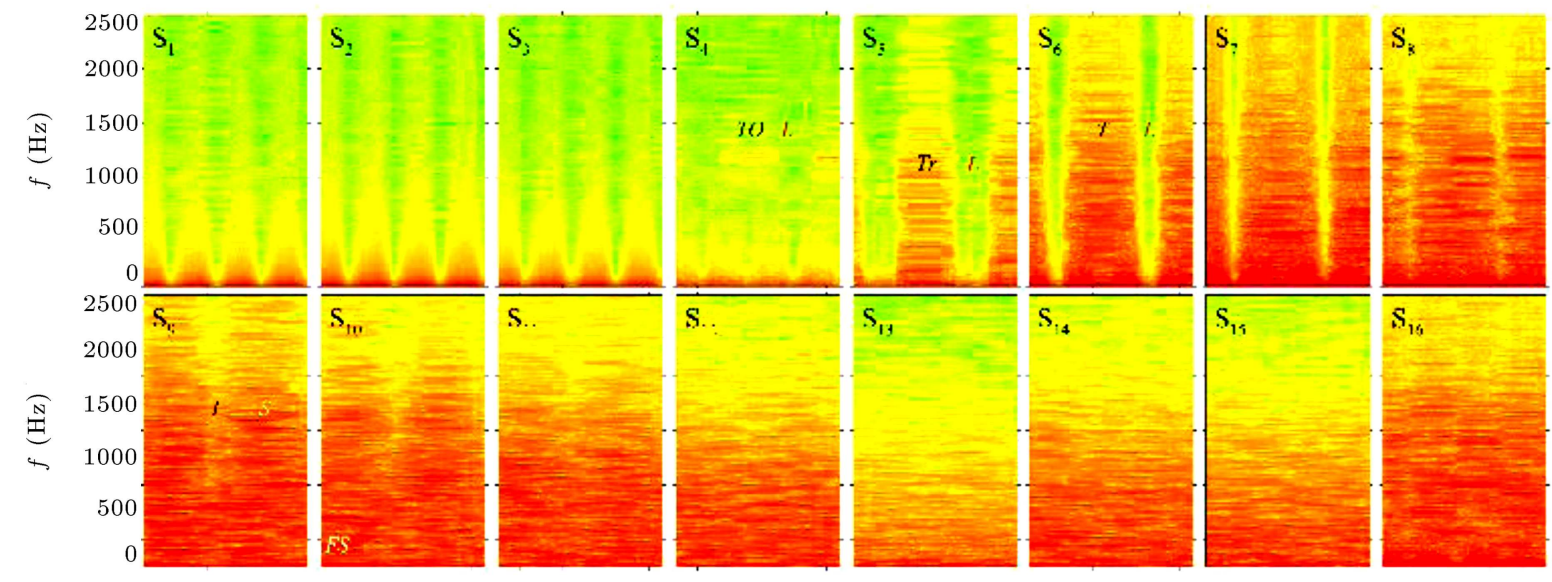

(i) $\kappa=0.1, \alpha_{m}=10 \mathrm{deg}$, and $\alpha_{a}=2 \mathrm{deg}$

Figure 8. Effects of oscillation parameters on the spectrogram of the hot-film outputs (continued).

may experience the separated flow during the entire oscillation cycle and are indicated by FS. According to Figure 8(1), several sensors may experience fully turbulent flow within the oscillation cycle and are marked by FT in the plots.

The flow fluctuations in time signals can be bet- ter understood by removing the low-frequency waves, especially those related to the body motion that can be performed through high-pass filtering of hot-film signals. To this end, this study considered a cut-off frequency of $10 \mathrm{~Hz}$. Figures 9 and 10 show the highpass filtered time signals for different oscillation cases. 

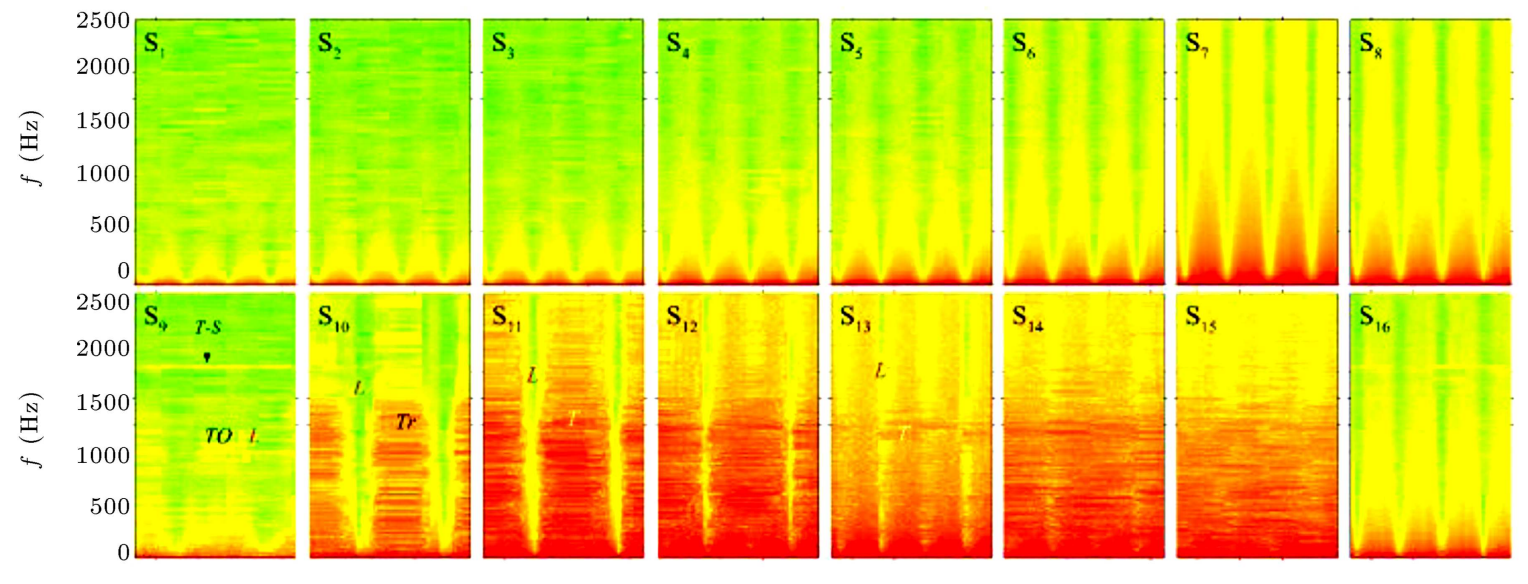

(j) $\kappa=0.1, \alpha_{m}=0 \mathrm{deg}$, and $\alpha_{a}=5 \mathrm{deg}$
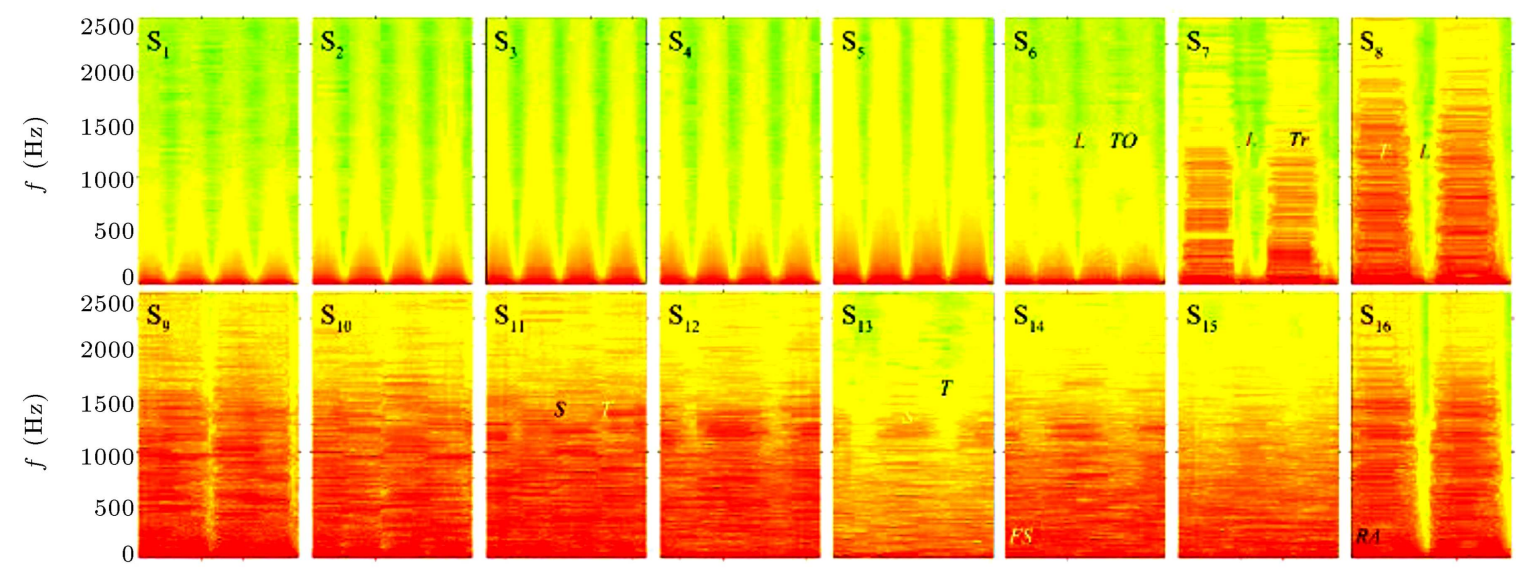

(k) $\kappa=0.1, \alpha_{m}=6 \mathrm{deg}$, and $\alpha_{a}=5 \mathrm{deg}$
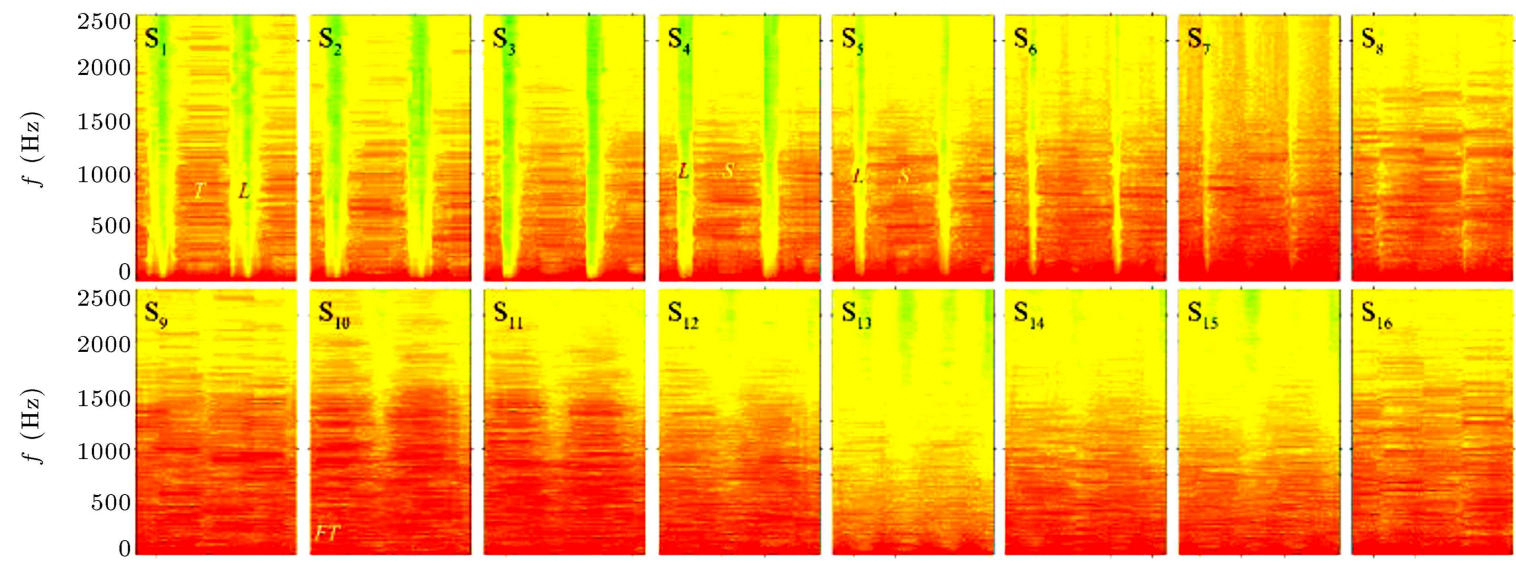

(1) $\kappa=0.1, \alpha_{m}=10 \mathrm{deg}$, and $\alpha_{a}=5 \mathrm{deg}$

Figure 8. Effects of oscillation parameters on the spectrogram of the hot-film outputs (continued).

A comparison between these figures and Figures 4 and 5 shows that the spatial/temporal domains of laminar, transitional, and turbulent flows could be simply recognized from the present figures, i.e., Figures 9 and 10. The spatial/temporal laminar flow regions could be detected as straight lines with approximately no signif- icant fluctuations. The transitional regions are those where fluctuations are significant and non-uniform. From Figures 9 and 10, one could conclude that an increase in the oscillation frequency may reduce the fluctuations. According to the results shown in these figures, as the mean angle increases from left to right, 

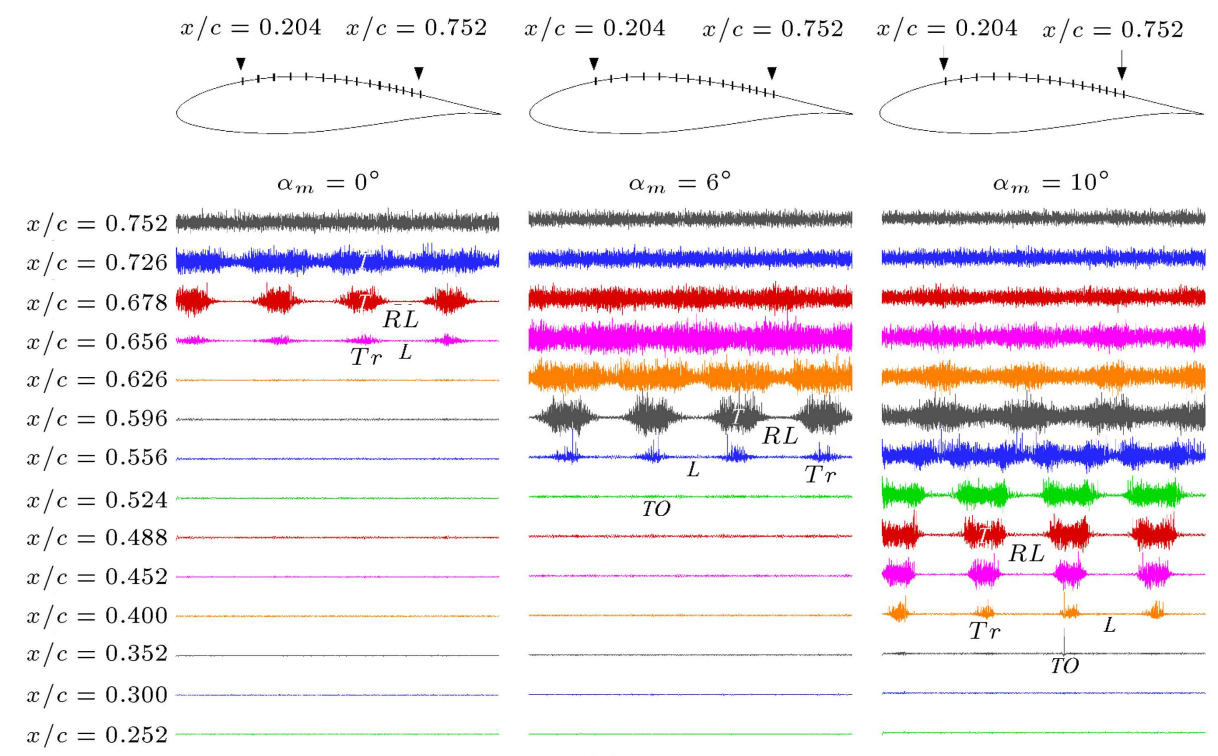

(a) $\kappa=0.04$

$$
\alpha_{m}=0^{\circ}
$$
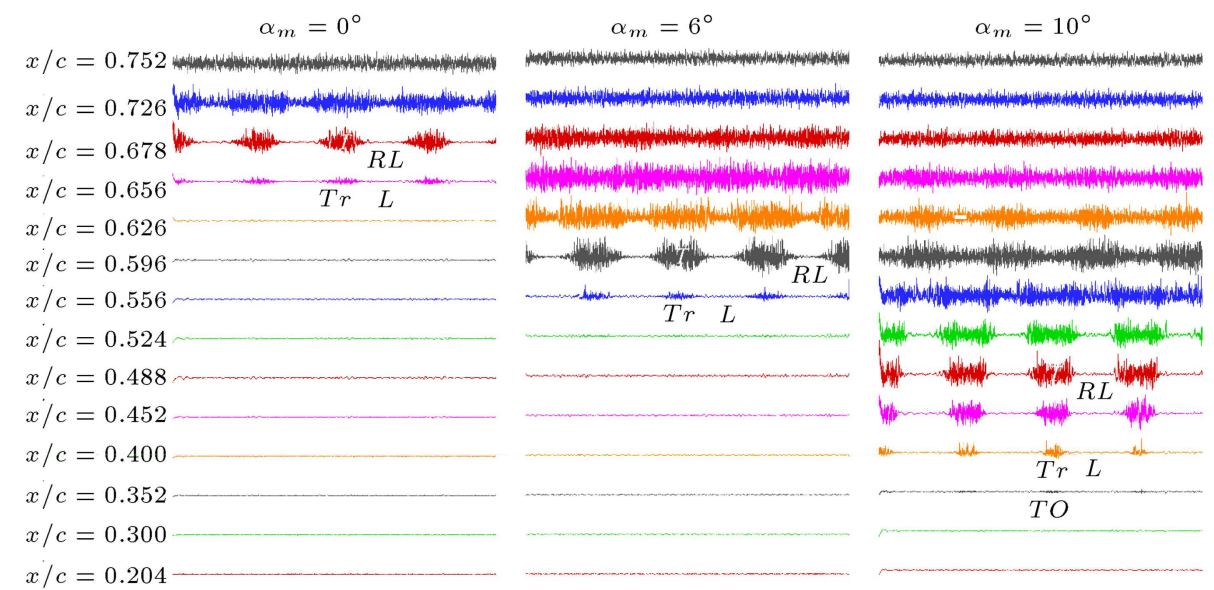

(b) $\kappa=0.10$

Figure 9. High-pass-filtered signals for oscillations with $\alpha_{a}=2 \mathrm{deg}$.

the number of hot-film sensors that experience transitional flow increases. This is also true for the effect of frequency amplitude. The spatial transitional flow regions are relatively wide for $\alpha_{a}=5 \mathrm{deg}$ (see Figure 10). The fully turbulent or separated flow regions are signals with uniform fluctuation distributions in time. The points of transition onset and relaminarization are well distinguished from the figures too.

From Figures 9 and 10, the hot-film signal could be decomposed as follows:

$$
E=E_{L P}+E_{H P}
$$

where the terms $E_{L P}$ and $E_{H P}$ correspond to the lowpass filtered and high-pass filtered parts of the signals, respectively. Using the time-frequency analysis, i.e., Fourier series over finite duration times, the high-pass filtered signals could be described as follows:

$$
E_{H P}(t)=\sum_{n=m}^{N_{p}} A_{n} \sin \left(2 \pi f_{n} t\right),
$$

where $N_{p}$ is the number of discrete points in the finite signal array, $f_{n}$ and $A_{n}$ are the frequency and amplitude of the $n$-th component wave, respectively, and $m$ is the index corresponding to the cut-off frequency. The unsteady characteristics of a hot-film signal regardless of the model motion are revealed through $E_{H P}(t)$. The RMS of a signal is a suitable means of cumulative oscillation amplitude measurement. This parameter describes the strength of an alternating signal. The RMS of Eq. (4) is given by Soltani et al. [22] as follows:

$$
E_{R M S}(t)=\frac{1}{\sqrt{2}} \sum_{n=k}^{N_{p}} A_{n} .
$$

Although $E_{R M S}$ is proportional to the summation of 

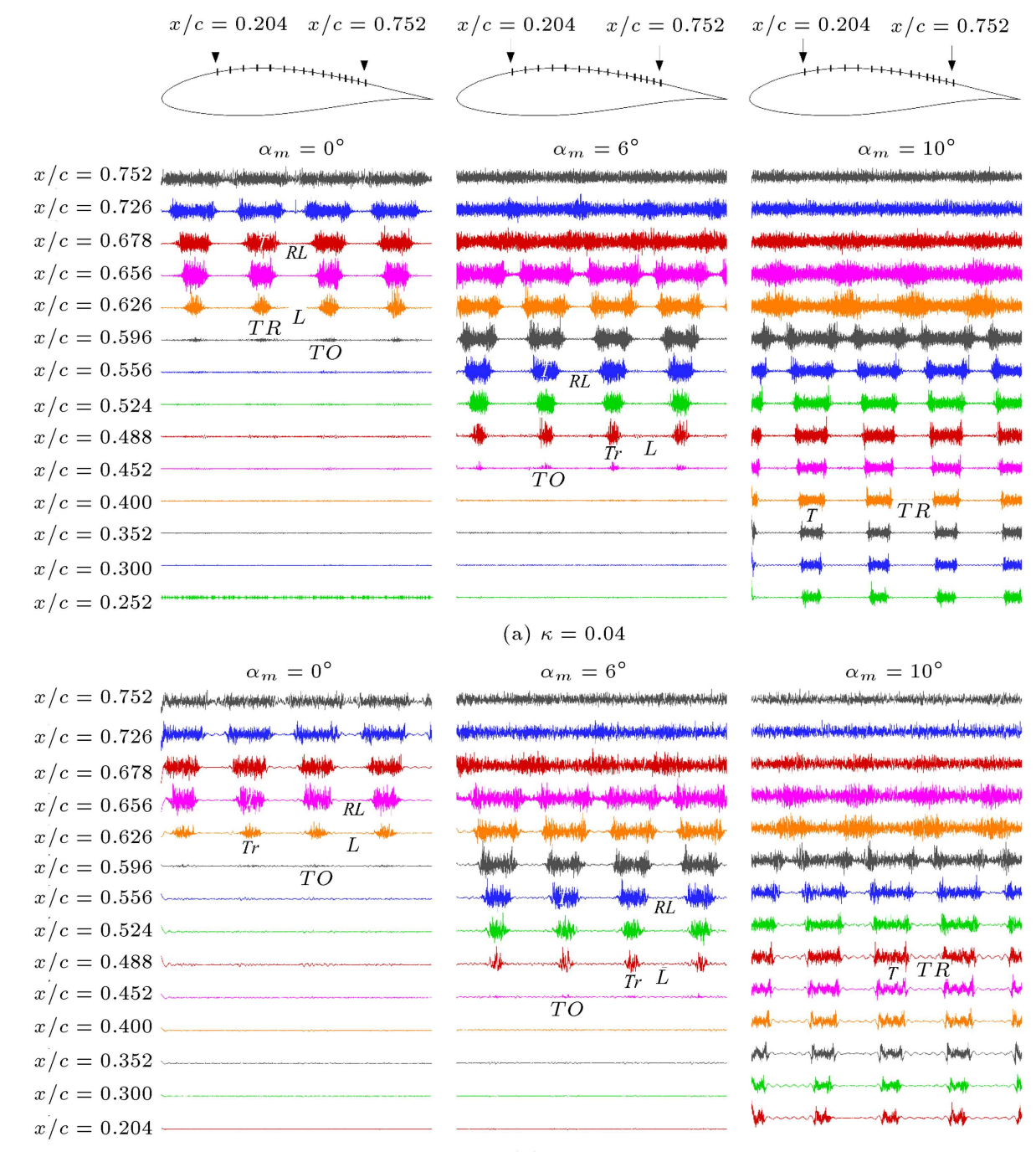

(a) $\kappa=0.04$
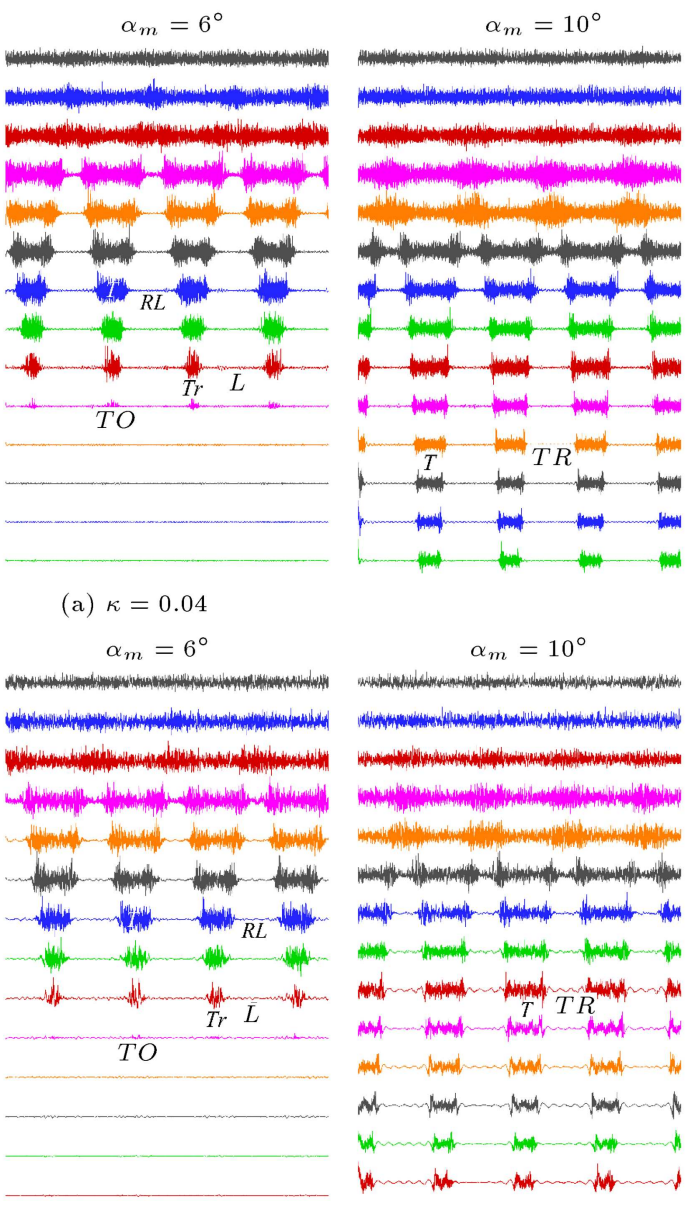

(b) $\kappa=0.10$

Figure 10. High-pass-filtered signals for oscillations with $\alpha_{a}=5 \mathrm{deg}$.

the wave amplitudes at different frequencies, it can be used as a sensor to detect the transition onset. The transition onset is where $E_{R M S}$ starts to rise significantly. Figure 11 shows the hot-film signal history, zero-mean high-pass filtered signal, and RMS distribution with respect to the pitch angle for an oscillating case with $\kappa=0.04, \alpha_{m}=6 \mathrm{deg}$, and $\alpha_{a}=5$ deg. Different BL events such as laminar, transitional, and turbulent temporal regions, transition onset, relaminarization point, and transition peak are detected and are shown in this figure based on the time-frequency analysis of the hot-film signals. In the laminar and turbulent flow situations, the RMS distribution is approximately uniform, while at the peak transition, the RMS has its maximum value (see Figure 11). Following the turbulent flow, as the pitching cycle further proceeds, the transitional flow occurs, which is followed by the relaminarization process. The temporal location of the turbulent flow takes place at the highest AOA, while the laminar flow occurs at the lowest.

Based on Eq. (5) and from the descriptions of Figure 11, one can determine the transition onset and relaminarization points in different oscillation cases. Figure 12 presents the variations of the transition point versus AOA for the current investigation. The data are compared with those obtained by the Xfoil code and, also, by the previous experimental studies using the same model $[15,18]$. The results are shown for the static condition, i.e., the angle of attack varies statically (pitch-pause motion). The transition points predicted by the Xfoil code for different amplification factors $\left(N_{\text {crit }}\right)$ are indicated by various lines. Different amplification factors correspond to different levels of turbulence in free stream flow. It could be seen that the present time frequency analysis results are in good agreement with the results of Ref. [18] and Xfoil prediction. 


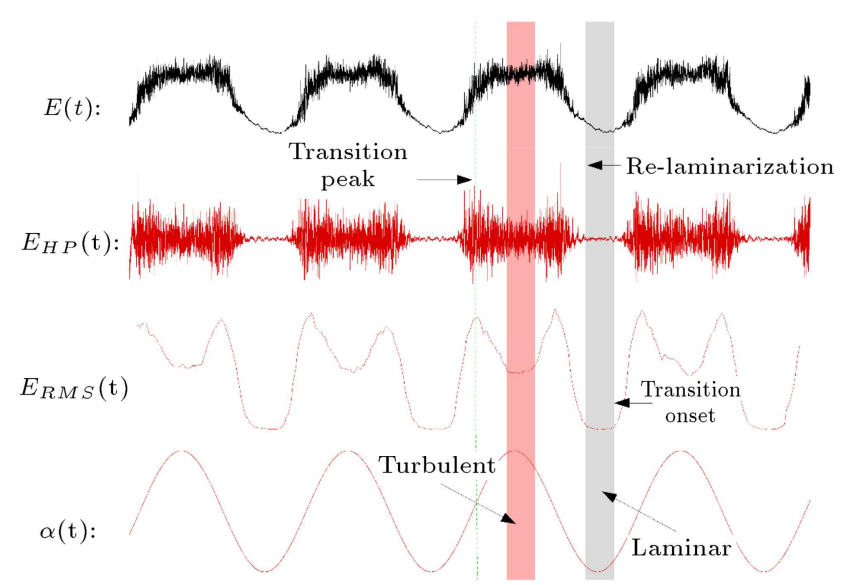

Figure 11. Original time signal, high-pass-filtered signal, Root-Mean-Squared (RMS), and angle of attack variations during pitching motion; $\kappa=0.04, \alpha_{m}=6 \mathrm{deg}$, and $\alpha_{a}=5$ deg.

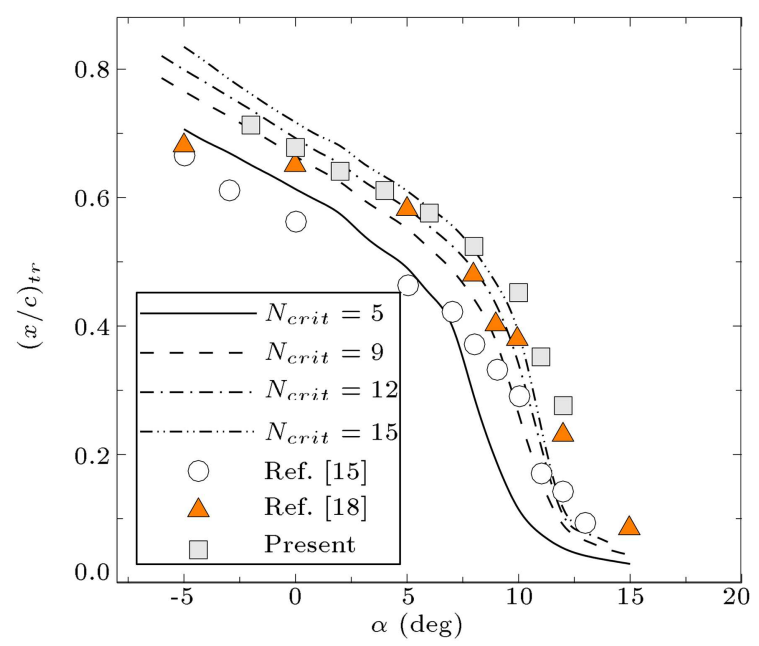

Figure 12. Spatial locations of the transition onset versus angle of attack for the static model.

The spatial locations for the transition onset and the relaminarization points are presented in Figure 13 for oscillation cases with $\kappa=0.1$ and $\alpha_{a}=5 \mathrm{deg}$. The measurement inaccuracy for the transition onsets is less than $5 \%$ in all test cases. The results correspond to the oscillation cases below (squares), within (triangles), and beyond (circles) the static stall AOA of the present model. The filled symbols are associated with the transition onset locations that occur during the pitchup and the open symbols indicate the relaminarization processes whose points take place during the pitchdown portion of the sinusoidal oscillation cycle. The solid line corresponds to the locations of the transition onset points for the static test obtained at a Reynolds number of $2.7 \times 10^{5}$. During the pitchup motion, the body motion improves the attachment of the flow over the surface which delays the transition onset with respect to the static condition, compared to the solid line with filled symbols in Figure 13. On the other hand, in

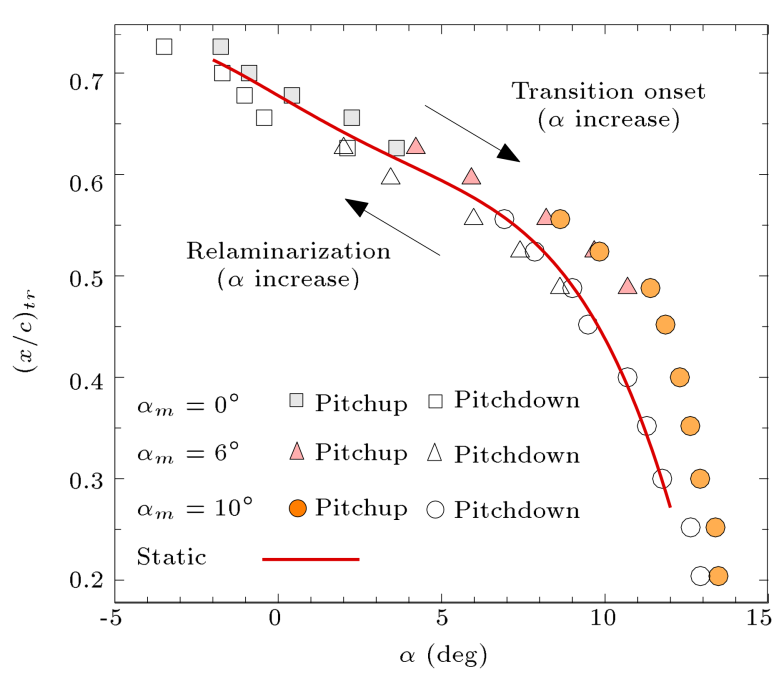

Figure 13. Spatial locations for transition onset and relaminarization points versus time-dependent angles of attack for oscillation cases with $\kappa=0.1$ and $\alpha_{a}=5 \mathrm{deg}$.

the pitchdown phase of the motion, the body motion promotes flow separation that enhances the transition. Hence, the relaminarization points during pitchdown occur at earlier spatial locations when compared to the transition onset for the static test. It could be observed that for cases $\alpha_{m}=0 \mathrm{deg}$ and $\alpha_{m}=6 \mathrm{deg}$, the transition onset locations for the static condition are between the transition onset locations for the pitchup and for the pitchdown conditions. According to Figure 13 , for the oscillation case with $\alpha_{m}=10 \mathrm{deg}$, the transition points for the static conditions are close to those for the pitchdown portion of the sinusoidal motion. For an airfoil oscillating beyond $\alpha_{s s}$, the BL events become more complicated. It is because of the growth and convection of an energetic Leading-Edge Vortex (LEV) that forms at high AOA [9]. The LEV for these oscillating cases improves the flow attachment that delays the transition onset.

Figure 14 investigates the effect of reduced frequency on the locations of the transition onset and on the relaminarization points for oscillating cases with $\alpha_{a}=5 \mathrm{deg}$ and $\kappa=0.1$. The results are presented for two cases of $\alpha_{m}=6 \mathrm{deg}$ and $\alpha_{m}=10 \mathrm{deg}$. Higher reduced frequency leads to higher hysteresis loop of the transition locations. Higher frequency of oscillation leads to higher acceleration of the body motion, which translates into further delay in the transition onset during the pitchup. This is similar to the effect of reduced frequency on the load hysteresis loop that has been previously reported $[9,15,16,19]$. Also, for oscillation beyond $\alpha_{s s}\left(\alpha_{m}=10 \mathrm{deg}\right)$, the hysteresis loop for transition onset locations is larger.

\section{Conclusion}

The time-frequency and space-frequency analyses of 


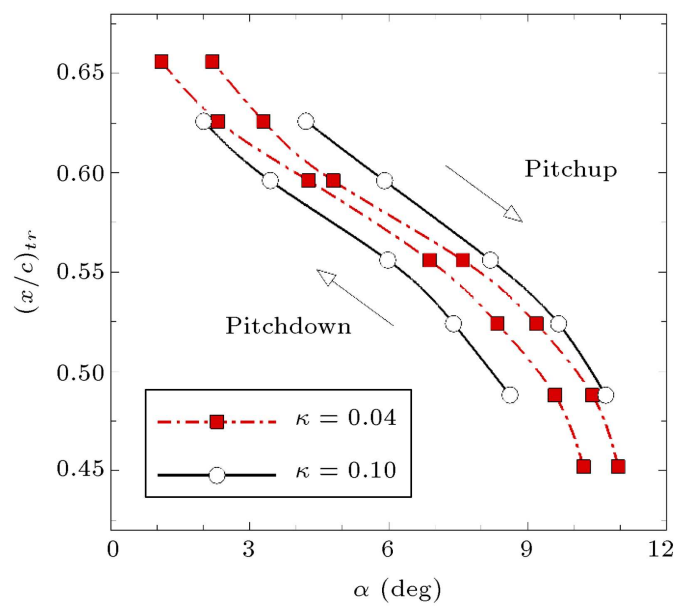

(a) $\alpha_{m}=6^{\circ}$

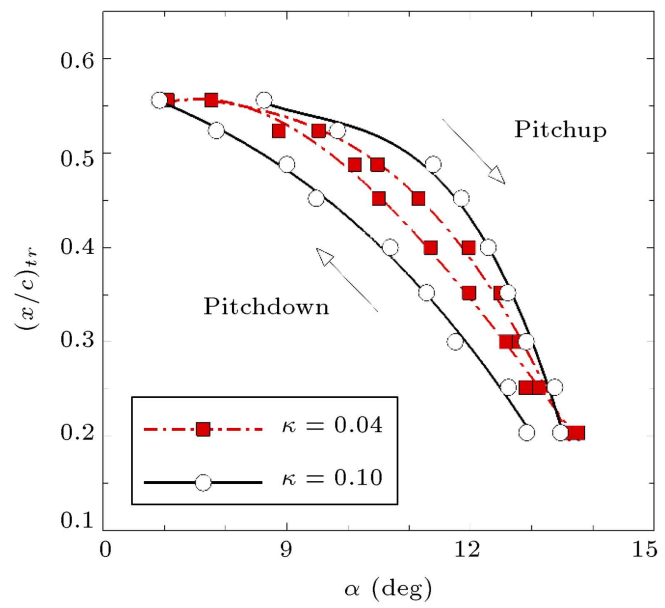

(b) $\alpha_{m}=10^{\circ}$

Figure 14. Effect of reduced frequency on the spatial locations of the transition onset and relaminarization points for oscillation cases with $\alpha_{a}=5 \mathrm{deg}$.

the hot-film data were carried out to interpret the Boundary Layer (BL) events over a pitching airfoil. To the authors' knowledge, these methods have not been used previously in the BL analysis, or if they have been used, the results and descriptions are not available. Laminar, transitional, and turbulent regions of BL are all detected in the space-frequency and time-frequency (spectrogram) plots. However, because of the unsteady motion of the model, the spatial/temporal locations of the BL events are tracked better from the spectrogram plots. The spectrogram plots introduced two precursor phenomena for the spatial/temporal locations of the turbulent flow and for the spatial location of the flow separation. Prior to the turbulent flow, regions of dominant high-frequency disturbances in spectrogram plots were developed. In addition, previous to the spatial regions with the fully separated flow, the pattern of spectrogram shows oscillation with a longer time period than the body motion oscillation. The flow then becomes fully separated during the oscillation cycle. A new technique based on the time-frequency analysis of the hot-film signals was presented to obtain both the transition onset and the relaminarization points. This technique functions based on the high-frequency fluctuations in the hot-film outputs. The results provided through the new technique are compared well with the experimental observations and previous investigations.

\section{Nomenclature}

$A \quad$ Wave amplitude

C Airfoil chord (m)

$E \quad$ Output voltage of hot-film sensor $(\mathrm{mV})$

$E_{0} \quad$ Offset voltage $(\mathrm{mV})$

$E_{H P} \quad$ High-pass filtered signal $(\mathrm{mV})$
$E_{L P} \quad$ Low-pass filtered signal $(\mathrm{mV})$

$E_{R M S} \quad$ A root-mean-squared parameter obtained from hot-film signal $(\mathrm{mV})$

$f \quad$ Wave frequency $(\mathrm{Hz})$

$f_{0} \quad$ Frequency for model oscillation $(\mathrm{Hz})$

Re Reynolds number based on the chord

$x \quad$ Spatial location along the chord $(\mathrm{m})$

$\alpha \quad$ Angle of attack (deg)

$\alpha_{a} \quad$ The amplitude of pitching oscillation (deg)

$\alpha_{m} \quad$ Mean angle of attack of pitching oscillation (deg)

$\alpha_{s s} \quad$ Stall angle of attack for static condition (deg)

$k \quad$ Reduced frequency of model oscillation

$\tau \quad$ Quasi-wall-shear stress

AOA Angle Of Attack

BL Boundary Layer

CTA Constant Temperature Anemometer

FS Fully Separated

FT Fully Turbulent

LEV Leading-Edge Vortex

LTT Laminar-to-Turbulent Transition

LSB Laminar Separation Bubble

QWSS Quasi-Wall Shear Stress

RA Reattached

RMS Root-Mean-Squared

TO Transition Onset

TS Tollmien-Schlichting 


\section{References}

1. Richter, K. and Schülein, E. "Boundary-layer transition measurements on hovering helicopter rotors by infrared thermography", Experiments in Fluids, 55(7), p. 1755 (2014).

2. Bellhouse, B.J. and Schultz, D.L. "Determination of mean and dynamic skin friction, separation and transition in low-speed flow with a thin-film heated element", Journal of Fluid Mechanics, 24(2), pp. 379400 (1966).

3. Stack, J., Mangalam, S., and Berry, S. "A unique measurement technique to study laminar-separation bubble characteristics on an airfoil", In 19th AIAA Fluid Dynamics, Plasma Dynamics, and Lasers Conference, AIAA 87-1271 (1987).

4. Nakayama, A., Stack, J., Lin, J., and Valarezo, W. "Surface hot-film method for the measurement of transition, separation and reattachment points", In 23rd Fluid Dynamics, Plasmadynamics, and Lasers Conference, p. 2918 (1993).

5. Hodson, H.P., Huntsman, I., and Steele, A.B. "An investigation of boundary layer development in a multistage LP turbine", Journal of Turbomachinery, 116(3), pp. 375-383 (1994).

6. Chandrasekhara, M.S. and Wilder, M.C. "Heat-flux gauge studies of compressible dynamic stall", AIAA Journal, 41(5), pp. 757-762 (2003).

7. Braune, M. and Koch, S. "Application of hot-film anemometry to resolve the unsteady boundary layer transition of a laminar airfoil experiencing limit cycle oscillations", 15th International Conference on Fluid Control, Measurements and Visualization, pp. 27-30 May, Naples, Italy (2019).

8. Lee, T. and Basu, S. "Measurement of unsteady boundary layer developed on an oscillating airfoil using multiple hot-film sensors", Experiments in Fluids, 25(2), pp. 108-117 (1998).

9. Lee, T. and Gerontakos, P. "Investigation of flow over an oscillating airfoil", Journal of Fluid Mechanics, 512, pp. 313-341 (2004).

10. Rudmin, D., Benaissa, A., and Poirel, D. "Detection of laminar flow separation and transition on a NACA0012 airfoil using surface hot-films", Journal of Fluids Engineering, 135(10), pp. 101-104 (2013).

11. Poels, A., Rudmin, D., Benaissa, A., and Poirel, D. "Localization of flow separation and transition over a pitching NACA0012 airfoil at transitional Reynolds numbers using hotfilms", Journal of Fluids Engineering, 137(12), p. 124501 (2015).

12. Richter, K., Koch, S., Gardner, A.D., Mai, H., Klein, A., and Rohardt, C.H. "Experimental investigation of unsteady transition on a pitching rotor blade airfoil", Journal of the American Helicopter Society, 59(1), p. 012001 (2014).
13. Gardner, A.D., Eder, C., Wolf, C.C., and Rafel, M. "Analysis of differential infrared thermography for boundary layer transition detection", Experiments in Fluids, 58(9), p. 122 (2017).

14. Gardner, A.D., Wolf, C.C., and Raffel, M. "Review of measurement techniques for unsteady helicopter rotor flows", Progress in Aerospace Sciences, 111, p. 100566 (2019).

15. Soltani, M.R. and Bakhshalipour, A. "Effect of amplitude and mean angle-of-attack on the boundary layer of an oscillating airfoil", The Aeronautical Journal, 112(1138), pp. 705-713 (2008).

16. Soltani, M.R., Seddighi, M., and Marzabadi, F.R. "Comparison of pitching and plunging effects on the surface pressure variation of a wind turbine blade section", Wind Energy, 12(3), pp. 213-239 (2009).

17. Soltani, M.R. and Marzabadi, F.R. "Experimental investigation of transition on a plunging airfoil", $S c i$ entia Iranica, Transaction B, Mechanical Engineering, 17(6), pp. 468-479 (2010).

18. Marzabadi, F.R. and Soltani, M.R. "Experimental study of the boundary layer over an airfoil in plunging motion", Acta Mechanica Sinica, 28(2), pp. 372-384 (2012).

19. Soltani, M.R., Marzabadi, F.R., and Mohammadi, Z. "Experimental study of the plunging motion with unsteady wind tunnel wall interference effects", $E x$ perimental Techniques, 36(5), pp. 30-45 (2012).

20. Popov, A.V., Botez, R.M., and Labib, M. "Transition point detection from the surface pressure distribution for controller design", AIA A Journal, 45(1), pp. 23-28 (2008).

21. Trapier, S., Deck, S., Duveau, P., and Sagaut, P. "Time-frequency analysis and detection of supersonic inlet buzz", AIAA Journal, 45(9), pp. 2273-2284 (2007).

22. Soltani, M.R., Daliri, A., Younsi, J.S., and Farahani, M. "Effects of bleed position on the stability of a supersonic inlet", Journal of Propulsion and Power, 32(5), pp. 1153-1166 (2016).

\section{Biographies}

Hassan Akhlaghi received $\mathrm{PhD}$ degree in Aerodynamics from Sharif University of Technology in 2021. His research interests include experimental Aerodynamics, unsteady Aerodynamics, turbomachinery, and rarefied gas dynamics.

Mohammad Reza Soltani received PhD degree in Applied Aerodynamics from University of Illinois, Urbana-Champaign, USA in 1991. He is a Professor at Aerospace Engineering Department at Sharif University of Technology, Iran. He is currently a Visiting Professor at William E. Boeing Department of Aeronautics and Astronautics at University of Washington, Seattle, USA. His research interests include 
unsteady aerodynamics, applied aerodynamics, design and building of wind tunnels and wind turbines, design, build, and implementation of wind tunnel instruments, and measurement methods.

Mohammad Javad Maghrebi received PhD degree in Mechanical Engineering from Monash University, Australia in 1999. He is currently a Professor at Mechanical Engineering Department at Ferdowsi University of Mashhad, Iran. His research interests include computational fluid dynamics, wind turbine, and heat transfer. 